\title{
Relationship between the Track and Structural Evolution of Hurricane Sandy (2012) Using a Regional Ensemble
}

\author{
ALEX M. KOWALESKI \\ Department of Meteorology and Atmospheric Science, The Pennsylvania State University, University Park, Pennsylvania \\ JENNI L. EVANS \\ Department of Meteorology and Atmospheric Science, and Institute for CyberScience, The Pennsylvania State University, \\ University Park, Pennsylvania
}

(Manuscript received 31 March 2018, in final form 11 October 2018)

\begin{abstract}
An ensemble of 72 Weather Research and Forecasting (WRF) Model simulations is evaluated to examine the relationship between the track of Hurricane Sandy (2012) and its structural evolution. Initial and boundary conditions are obtained from ECMWF and GEFS ensemble forecasts initialized at 0000 UTC 25 October. The 5-day WRF simulations are initialized at 0000 UTC 27 October, $48 \mathrm{~h}$ into the global model forecasts. Tracks and cyclone phase space (CPS) paths from the 72 simulations are partitioned into 6 clusters using regression mixture models; results from the 4 most populous track clusters are examined. The four analyzed clusters vary in mean landfall location from southern New Jersey to Maine. Extratropical transition timing is the clearest difference among clusters; more eastward clusters show later Sandy-midlatitude trough interaction, warm seclusion formation, and extratropical transition completion. However, the intercluster variability is much smaller when examined relative to the landfall time of each simulation. In each cluster, a short-lived warm seclusion forms and contracts through landfall while lower-tropospheric potential vorticity concentrates at small radii. Despite the large-scale similarity among the clusters, relevant intercluster differences in landfall-relative extratropical transition are observed. In the easternmost cluster the Sandytrough interaction is least intense and the warm seclusion decays the most by landfall. In the second most eastward cluster Sandy retains the most intact warm seclusion at landfall because of a slightly later (relative to landfall) and weaker trough interaction compared to the two most westward clusters. Nevertheless, the remarkably similar large-scale evolution of Sandy among the four clusters indicates the high predictability of Sandy's warm seclusion extratropical transition before landfall.
\end{abstract}

\section{Introduction}

During the four days before its devastating New Jersey landfall at 2330 UTC 29 October 2012, interactions between Hurricane Sandy (2012) and two troughs governed the structural evolution of the hurricane (Blake et al. 2013). On 26 and 27 October, a subtropical uppertropospheric trough over the eastern Gulf of Mexico initiated the extratropical transition (ET) of Sandy. On 29 and 30 October, a larger midlatitude trough over the eastern United States steered Sandy toward landfall. This second interaction also caused Sandy to acquire warm seclusion characteristics before completing ET (Galarneau et al. 2013). While undergoing this second trough

\footnotetext{
Corresponding author: Jenni L. Evans, jle7@psu.edu
}

interaction, Hurricane Sandy made landfall as an extratropical cyclone with maximum sustained wind speed (minimum pressure) of $36 \mathrm{~m} \mathrm{~s}^{-1}$ ( $\left.945 \mathrm{hPa}\right)$. The increase in Sandy's size and intensity resulting from these the two trough interactions contributed to the massive storm surge at landfall, which was responsible for the majority of the 72 fatalities and the estimated $\$ 50$ billion (2012 U.S. dollars) in damage in the United States (Blake et al. 2013).

Previous studies of tropical cyclone-trough interaction have demonstrated that tropical cyclone (TC) intensity evolution is highly sensitive to the relative position and/ or structure of interacting troughs. Hanley et al. (2001) found that favorable distant TC-trough interactions tend to involve a weaker upper-level potential vorticity (PV) trough than unfavorable interactions. They argued that in favorable interactions, enhanced upper-level divergence 
from the trough outweighs the negative effects of increased vertical wind shear. Conversely, Kimball and Evans (2002) used idealized numerical simulations to show that TC intensification is most favored when the interacting upper-level PV trough is intense, but vertically shallow. More recently, Leroux et al. (2016) investigated how the evolution of Cyclone Dora (2007) varied with changes in its initial trough-relative position. Their simulations showed that $1^{\circ}-4^{\circ}$ changes in Dora's initial position substantially affected the subsequent structural evolution of the trough, and whether Dora underwent a favorable or unfavorable interaction.

The sensitivity of TC evolution during and after ET to trough position and structure is particularly well documented. Klein et al. (2002) varied the initial TC position of two western North Pacific ET events and found that these variations greatly affected the TC-trough interaction, and thus the ex-TC's post-ET intensity evolution. The idealized simulations of Ritchie and Elsberry (2003, 2007) showed that a stronger midlatitude trough produced less TC weakening during ET, while TCs initially closer to a midlatitude trough reintensified more after ET completion. Composites of North Atlantic ET events produced by Hart et al. (2006) demonstrated that ex-TCs that reintensify (weaken) after ET tend to approach more closely to (remain farther from) a negatively tilted (positively tilted) midlatitude trough. A negatively tilted trough produces less shear over the TC in advance of the upper(trough) and lower-tropospheric (TC) PV interaction, and drives greater eddy PV fluxes into the TC. Milrad et al. (2009) confirmed that a negatively tilted trough favors intensification of TCs affecting eastern Canada; they attributed the intensification to a feedback loop between latent heat release, downstream ridge building, reduction of the trough-ridge wavelength, and increased cyclonic vorticity advection over the surface cyclone.

Hart et al. (2006) and Kofron et al. (2010) found that the structure of the interacting trough is important in determining whether an ex-TC develops a warm seclusion structure or remains cold core after ET. Ex-TCs that develop a warm seclusion structure interact with narrower, but vertically deeper troughs than ex-TCs that remain cold core. Hart et al. (2006) contended that a narrower and deeper trough promotes scale matching between the trough and ex-TC and focuses the eddy momentum flux in the ex-TC's outflow layer. This disrupts thermal wind balance and enhances the cyclone's secondary circulation, which promotes intensification and warm seclusion formation. Notably, however, Hart et al. (2006) discussed warm seclusion formation after ET completion, whereas the seclusion of Sandy's warm core occurred during ET (Galarneau et al. 2013; Blake et al. 2013).
Galarneau et al. (2013) used a convection-permitting Weather Research and Forecasting (WRF) Model simulation to document how the interaction between Sandy and the trough over the eastern United States led to intensification and warm seclusion formation. Lowertropospheric baroclinicity increased as cool air from the trough partially encircled Sandy's core, enhancing Sandy's secondary circulation and causing intensification. Furthermore, a frontogenesis axis along the Gulf Stream generated substantial lower-tropospheric PV late on 28 October and early on 29 October. As the secondary circulation strengthened and the warm seclusion contracted, this PV was axisymmetrized into Sandy's inner core, contributing to intensification.

Forecasts of Hurricane Sandy from various ensemble prediction systems (EPSs), especially those initialized prior to 27 October, yielded substantial spread in storm track (Magnusson et al. 2014). While the European Centre for Medium-Range Weather Forecasts' Integrated Forecast System (ECMWF IFS) predicted the northwest turn and landfall of Sandy more skillfully than the National Centers for Environmental Prediction's Global Ensemble Forecast System (NCEP GEFS) at 58-day lead times, both ensembles showed considerable spread early in Sandy's life cycle. Kowaleski and Evans (2016) performed regression mixture-model path clustering (Kuruppumullage Don et al. 2016) on forecasts from four global EPSs to demonstrate a relationship between track and storm structure as indicated by cyclone phase space (CPS; Hart 2003; Evans and Hart 2003) for initialization times from 23 to 27 October. Track clusters in which Sandy moved farther east before turning northwest were associated with later trough interaction and ET completion.

Results from Munsell and Zhang (2014) also suggest that variations in the track of Sandy affected the storm's evolution during ET. Although that study focused on the cause of track differences among a 60-member WRF ensemble, it also found that simulations that accurately represented Sandy's track yielded maximum stormtrough interaction just before landfall. In contrast, simulations in which Sandy tracked farther eastward showed maximum interaction earlier relative to landfall, when Sandy was farther from the trough axis. This more distant interaction produced less TC intensification (as measured by lower-tropospheric PV) and less precipitation over land.

Here, we extend the methodology of Kowaleski and Evans (2016) to investigate the processes governing the relationship between the track and structural evolution of Hurricane Sandy during ET. Convection-permitting WRF simulations are generated using IFS and GEFS forecasts initialized at 0000 UTC 25 October. The tracks 


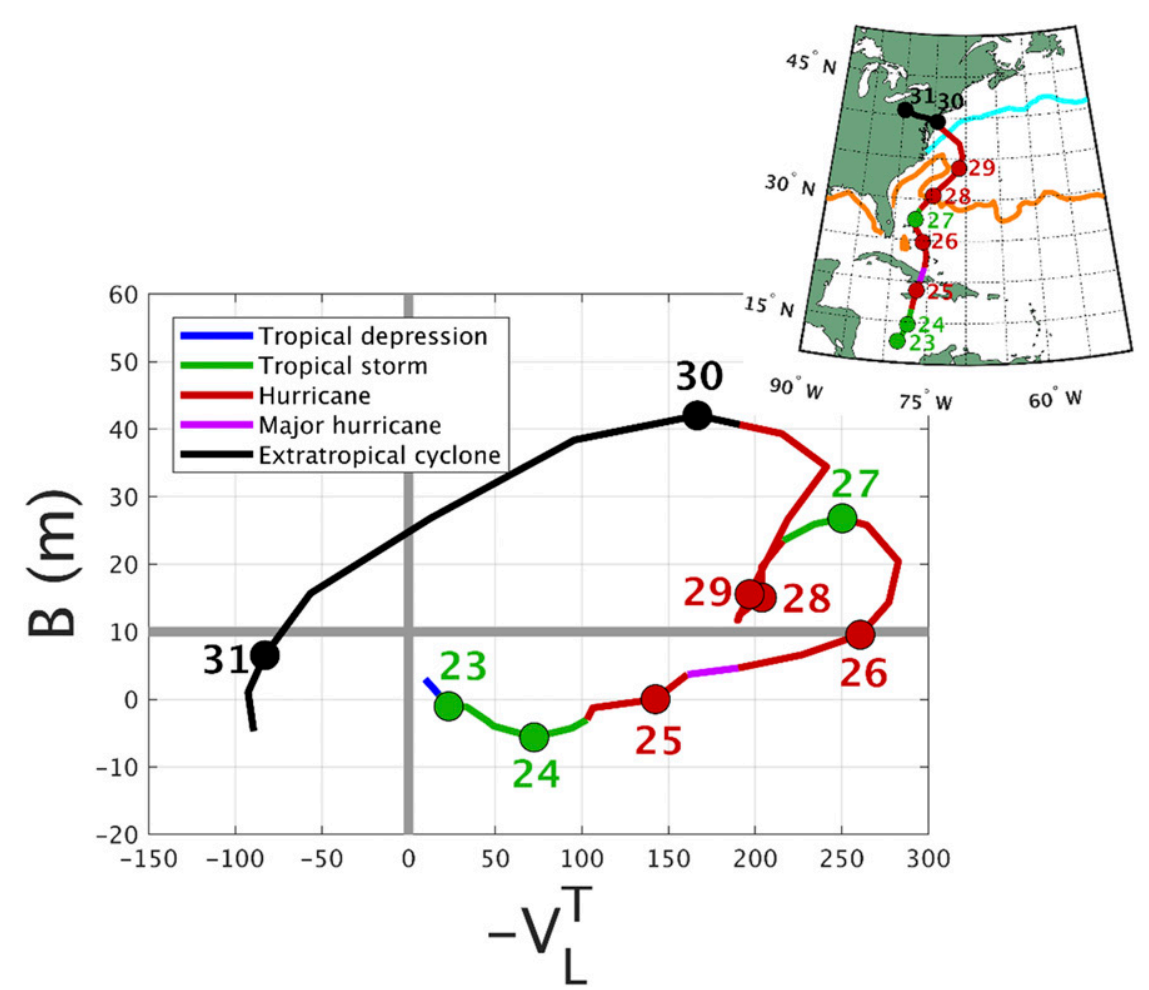

FIG. 1. Cyclone phase space (CPS) evolution of Hurricane Sandy derived from the ECMWF analysis (obtained at $0.14^{\circ}$ horizontal resolution; ECMWF 2011). The National Hurricane Center (NHC) best track of Sandy is shown in the inset; the $26^{\circ} \mathrm{C}\left(20^{\circ} \mathrm{C}\right)$ sea surface temperature isotherm at 0000 UTC 29 Oct is contoured in orange (cyan) for reference. Labeled dots indicate the structure and location (inset) of Sandy at 0000 UTC each day. Storm classifications are from the NHC best track of Sandy (Blake et al. 2013). All CPS data shown in this paper are computed from a 24-h running mean.

of Sandy among the WRF simulations are clustered using regression mixture models; results from the four most populous clusters are analyzed to show how the structural evolution of Sandy varies with storm track.

This paper proceeds as follows: a brief overview of the synoptic evolution of Sandy provides context for the results documented here and previous findings (section 2), description of the WRF ensemble (section 3), selection of optimal cluster specifications (section 4), and examination of results (section 5). Results are summarized, and conclusions presented in section 6 .

\section{Overview of the synoptic evolution of Hurricane Sandy}

The tropical depression that became Hurricane Sandy formed in the south-central Caribbean Sea at 1200 UTC 22 October 2012 and reached tropical storm strength six hours later. On 23 October, Sandy began to move north-northeastward while intensifying (Fig. 1). A digging upper-level trough northwest of Sandy accelerated the TC's north-northeast motion, beginning around 1200 UTC
23 October (Blake et al. 2013). As Sandy accelerated, it continued to intensify, becoming a hurricane at 1200 UTC 24 October. Sandy made its first landfall in eastern Jamaica seven hours later with maximum winds of $39 \mathrm{~m} \mathrm{~s}^{-1}(75 \mathrm{kt})$. After transiting Jamaica, Sandy continued to move north-northeastward and intensified further, becoming a category 3 major hurricane $\left(51 \mathrm{~m} \mathrm{~s}^{-1}\right.$; $100 \mathrm{kt}$; Fig. 1) just before landfall near Santiago, Cuba, at 0525 UTC 25 October (Blake et al. 2013).

Passage over Cuba caused Sandy to weaken to a category 2 storm and increased southwesterly shear from the upper-level trough caused continued weakening through 25 and 26 October. On 26 October, Sandy began to undergo ET as the interaction between the TC and trough continued; the definition of ET onset follows that of Evans and Hart (2003), where Sandy's across-track 900-600-hPa thickness difference $B$ exceeded $10 \mathrm{~m}$ (Fig. 1). This increase in baroclinicity was associated with the development of a warm (stationary) front in Sandy's northeast (northwest) quadrant. During this trough interaction, Sandy weakened to a tropical storm by 0000 UTC 27 October, while its wind field expanded greatly (Blake et al. 2013). 

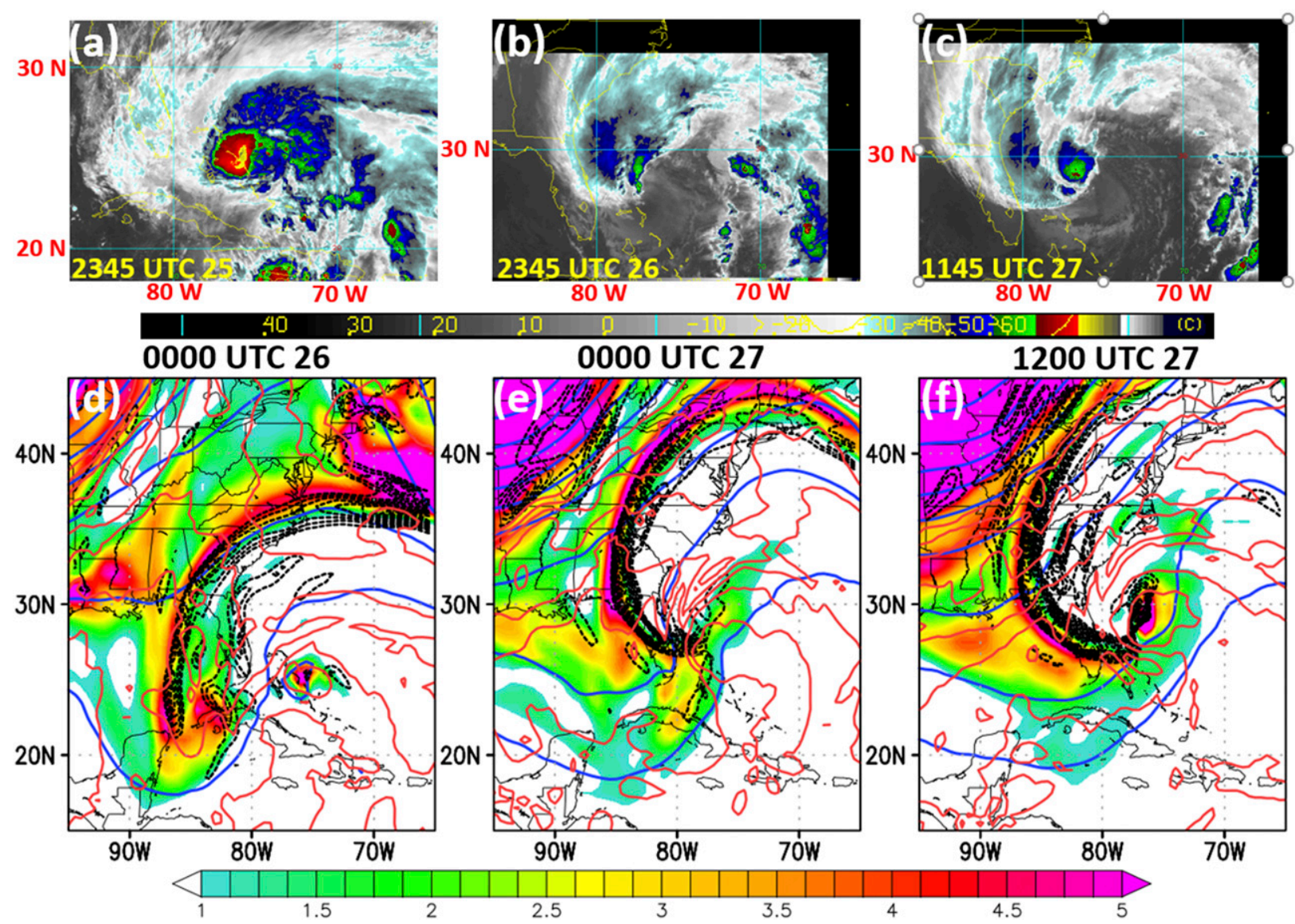

FIG. 2. CIRA Regional and Mesoscale Meteorology Branch (RAMMB) 4-km enhanced infrared satellite imagery of Sandy at (a) 2345 UTC 25 Oct, (b) 2345 UTC 26 Oct, and (c) 1145 UTC 27 Oct, along with NCEP GFS $0.5^{\circ}$ analyses depicted at (d) 0000 UTC 26 Oct, (e) 0000 UTC 27 Oct, and (f) 1200 UTC 27 Oct. (d)-(f) Potential vorticity on the 345-K isentrope (PVU), 345-K PV advection by the divergent wind (dashed black contours every $-4 \mathrm{PVU} \mathrm{day}^{-1}$ from -4 to $-48 \mathrm{PVU}$ day $^{-1}$ ), 250-hPa geopotential height (blue contours every $90 \mathrm{~m}$ ), and 700 -hPa potential temperature (red contours every $2 \mathrm{~K}$ from 300 to $314 \mathrm{~K}$ ).

Although Sandy retained a strong low-level warm core $\left(-\mathbf{V}_{L}^{T}>200\right)$ on 26 and 27 October (Fig. 1), convection became concentrated northwest of the center (cf. Figs. 2b and 2a), in the region of enhanced baroclinicity associated with the stationary front (Fig. 2e; Galarneau et al. 2013). Outflow from this convection produced negative PV advection, halting the trough's eastward progression and facilitating cyclonic wave breaking, leading Sandy to track more westward (Figs. 2d-f; Galarneau et al. 2013; Torn et al. 2015). The increasingly negative tilt of the trough reduced vertical wind shear over Sandy, and the storm regained hurricane strength around 1200 UTC 27 October as convection redeveloped near its center (Fig. 2c; Blake et al. 2013). On 27 and 28 October, ET halted, as shown by the decrease in thickness asymmetry during this period (Fig. 1). Although low-level baroclinicity decreased as Sandy moved away from the upper-level trough, Sandy retained an expansive wind field because of the trough interaction, with a radius of maximum winds exceeding $130 \mathrm{~km}$ (Blake et al. 2013; Demuth et al. 2006).

Early on 29 October, Sandy turned north under the influence of an anomalous midlevel ridge to its northeast (Fig. 3c; Blake et al. 2013). Concurrently, a cyclonic Rossby wave breaking event caused a large midtropospheric trough to move into the southeast United States and acquire a substantial negative tilt (Figs. 3d,e; Blake et al. 2013; Torn et al. 2015). The negative tilt of the trough promoted reintensification of Sandy as a result of reduced vertical wind shear and enhanced upper-level divergence, while steering Sandy northwestward toward land (Blake et al. 2013). During this period, cool continental air partially encircled Sandy's center, forming a short-lived warm seclusion that contributed to intensification (Fig. 3d; Galarneau et al. 2013). As Sandy passed over the Gulf Stream, it attained a secondary peak in maximum sustained wind 

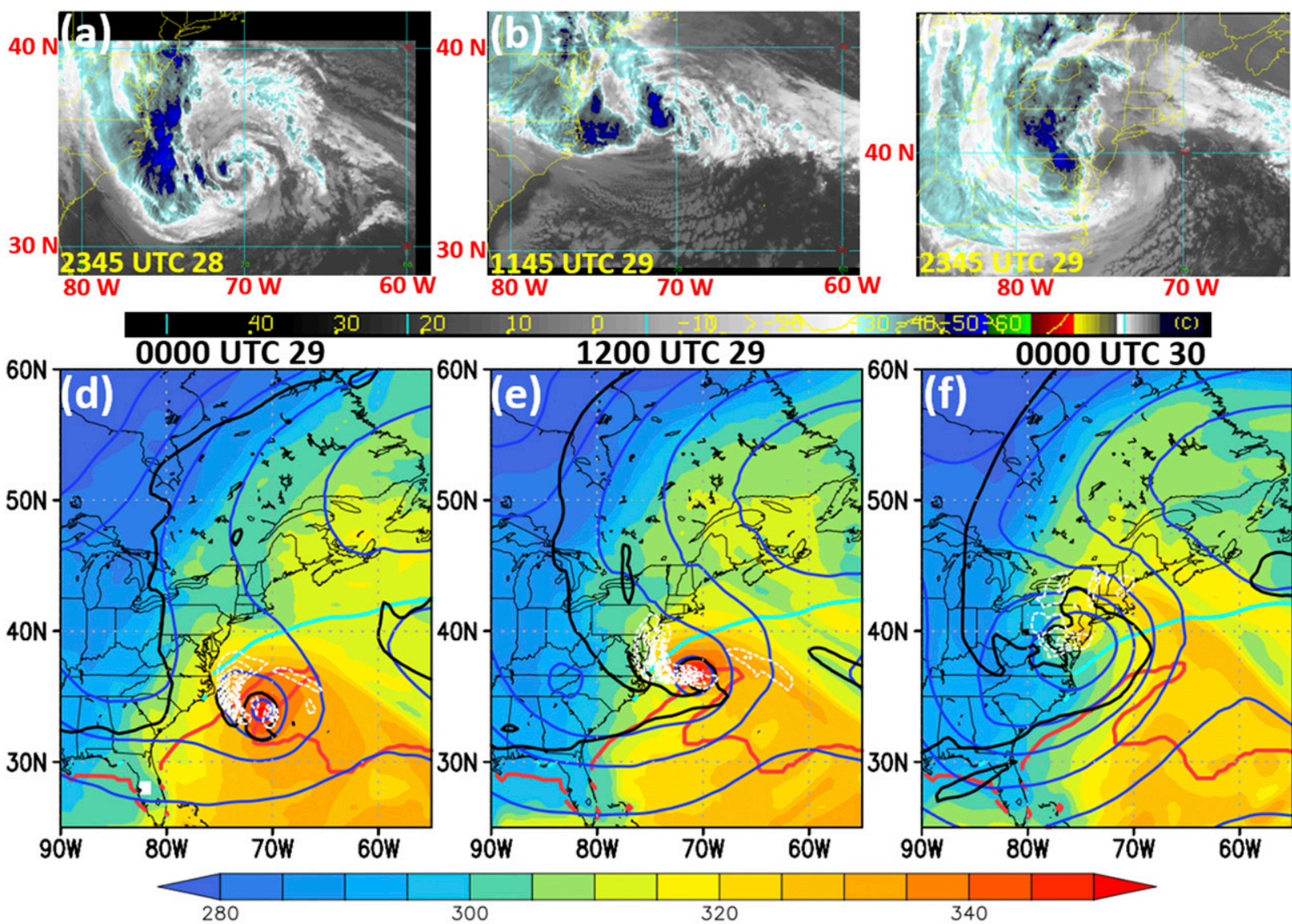

FIG. 3. CIRA Regional and Mesoscale Meteorology Branch (RAMMB) 4-km enhanced infrared satellite imagery of Sandy at (a) 2345 UTC 28 Oct, (b) 1145 UTC 29 Oct, and (c) 2345 UTC 29 Oct, along with NCEP GFS $0.5^{\circ}$ analyses depicted at (d) 0000 UTC 29 Oct, (e) 1200 UTC 29 Oct, and (f) 0000 UTC 30 Oct. (d)-(f) The 850-hPa equivalent potential temperature (K; shaded), 500-hPa geopotential height (blue contours every $90 \mathrm{~m}$ ), the 325-K 2-PVU contour (black), and integrated 850-200-hPa upward water vapor flux $\left(\mathrm{kg} \mathrm{m}^{-1} \mathrm{~s}^{-1}\right.$; dashed white contours every $3 \mathrm{~kg} \mathrm{~m}^{-1} \mathrm{~s}^{-1}$ from 3 to $36 \mathrm{~kg} \mathrm{~m}^{-1} \mathrm{~s}^{-1}$ ), and SST (red and cyan contours at $26^{\circ}$ and $20^{\circ} \mathrm{C}$ ).

speed $\left(44 \mathrm{~m} \mathrm{~s}^{-1} ; 85 \mathrm{kt}\right)$ at 1200 UTC 29 October, reaching its lowest central pressure $(940 \mathrm{hPa})$ six hours later (Blake et al. 2013).

After 1500 UTC 29 October, Sandy moved north of the Gulf Stream over substantially lower sea surface temperatures (SSTs; Fig. 3f). During this time, cool air from the midlatitude trough reached the inner core of Sandy. These factors reduced inner-core convection, hastening ET completion (Figs. 1 and 3c,f). Based on the National Hurricane Center (NHC) best track, the storm became extratropical at 2100 UTC 29 October ( $2.5 \mathrm{~h}$ before landfall; Blake et al. 2013) as inner-core convection collapsed and surface fronts reached Sandy's center. However, based on the criterion of Evans and Hart $\left(2003 ; \mathbf{V}_{L}^{T}<0\right)$, Sandy did not complete ET until after landfall, on 30 October (Fig. 1), as the lower-tropospheric warm core persisted for a short time after the NHC designated Sandy extratropical. After making landfall, Sandy moved west-northwestward and weakened, becoming ill defined after 1200 UTC 31 October (Blake et al. 2013).

\section{Data and development of the WRF ensemble}

A total of 72 global ensemble simulations from the ECMWF IFS (50 ensembles + control) and NCEP GEFS (20 ensembles + control), initialized at 0000 UTC 25 October, are used to initialize the convectionpermitting WRF simulations examined here. The WRF simulations have higher horizontal resolution than the global ensembles as well as higher temporal resolution of output data. Thus, the WRF ensemble provides higher-resolution representations of processes involving Sandy's core and storm-trough interaction before landfall.

GEFS ensemble forecasts are obtained from the National Centers for Environmental Information (NCEI; NCEI 2018) at a $1^{\circ}$ horizontal resolution and 25 pressure 

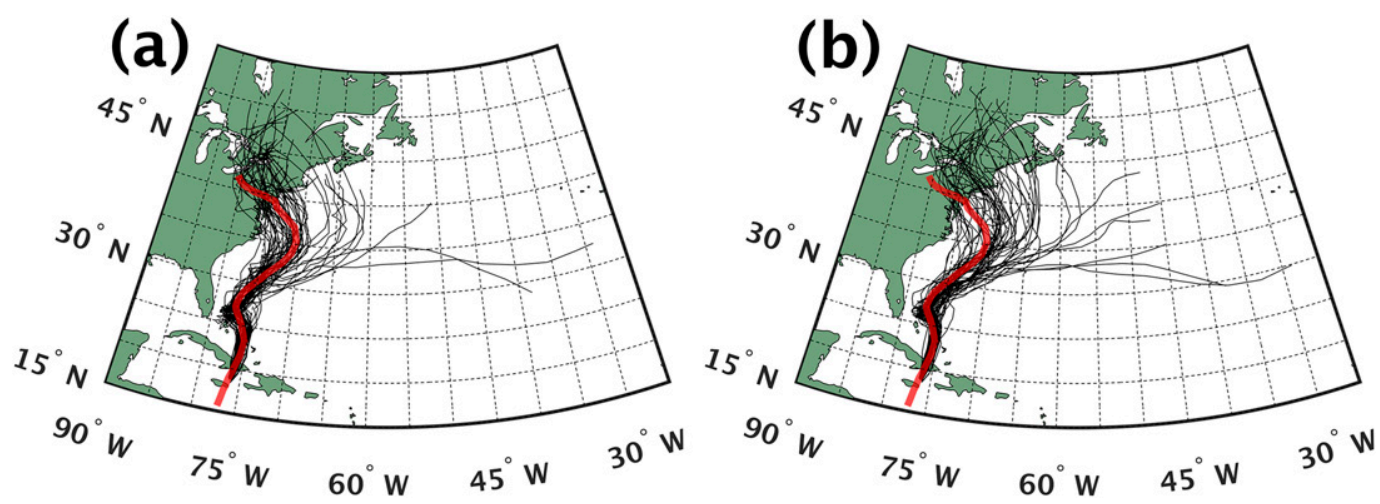

FIG. 4. Forecast tracks of Sandy initialized at 0000 UTC 25 Oct from (a) the 51-member ECMWF IFS operational at the time of Sandy (Cycle 38r1), and (b) the ECMWF IFS operational in 2016 (Cycle 41r2). Tracks are generated using the GFDL vortex tracker (Marchok 2002). The best track of Sandy in each panel is shown in red.

levels. IFS ensemble forecasts are obtained at $0.25^{\circ}$ horizontal resolution and 91 model levels. Because ECMWF does not archive data at the vertical resolution required to initialize WRF simulations (L. Magnusson 2016, personal communication), the IFS simulations employed in this study are generated from a retrospective run of the 2016 IFS (Cycle 41r2; ECMWF 2017), rather than the version operational in October 2012 (Cycle 38r1). Cycle 41r2 has approximately 18-km horizontal resolution and 91 vertical levels, compared to $32-\mathrm{km}$ horizontal resolution and 62 vertical levels in Cycle 38r1. The retrospective run using Cycle 41r2 produces a comparable track distribution to the operational ensemble, though with a somewhat greater spread (cf. Figs. $4 \mathrm{a}$ and $4 \mathrm{~b}$ ).

Each global ensemble member provides initial and boundary conditions for a corresponding simulation of Sandy using WRF, version 3.8 (Skamarock et al. 2008). Simulations are two way triple nested, with two vortexfollowing nests centered on Sandy, and 4-km resolution in the innermost nest (Fig. 5). Updated lateral boundary conditions for the outermost WRF domain are provided from the global simulation every six hours. ${ }^{1}$ While the global ensembles are initialized at 0000 UTC 25 October, the WRF simulations are initialized at 0000 UTC 27 October, because initializing WRF simulations at 0000 UTC 25 October yields insufficient track spread. Additional information on the WRF configuration is found in Table 1.

The ensemble of WRF simulations produces somewhat less spread than the multi-EPS global ensemble

\footnotetext{
${ }^{1}$ For GEFS ensemble members 06 and 08 , some forecast times have missing data, so their corresponding WRF simulations have updated lateral boundary conditions every 18 and $12 \mathrm{~h}$, respectively.
}

from which it was derived (cf. Figs. 6a and 6b). The lack of stochastic physics among the WRF simulations may contribute to this smaller spread. Among the WRF ensemble, fewer simulations have Sandy moving out to sea, compared to the global ensemble. The WRF ensemble contains more members in which Sandy makes landfall near the observed landfall location; however, it still has sufficient spread to provide insights into how Sandy's structural evolution varies with storm track.

\section{Selection of optimal cluster specifications}

The 72 WRF simulations (section 3) are partitioned into clusters by geographic track and CPS path of Hurricane Sandy. This clustering employs the regression mixture-modeling implemented for this purpose by

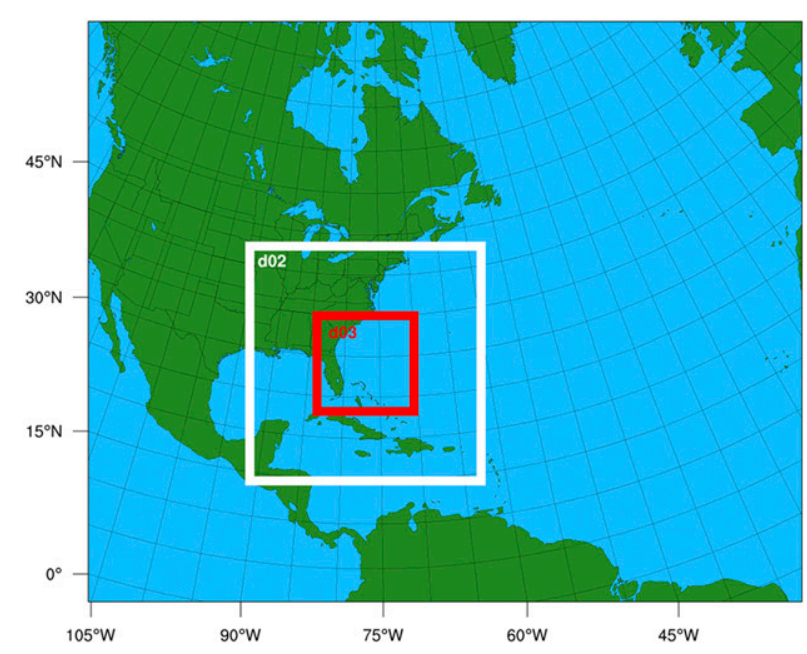

FIG. 5. WRF domains used in all simulations of Hurricane Sandy. The initial positions of the inner two domains change slightly among simulations based on the position of Sandy at 0000 UTC 27 Oct in the global ensemble member used to initialize WRF. 
TABLE 1. Model configuration used in WRF, version 3.8, simulations of Hurricane Sandy. Model domains are depicted in Fig. 5. Settings used in all three domains are only listed in the domain 1 column. Further information on the selected settings can be found in Skamarock et al. (2008).

\begin{tabular}{|c|c|c|c|}
\hline Model parameter & Domain 1 & Domain 2 & Domain 3 \\
\hline Horizontal grid spacing $(\mathrm{km})$ & 36 & 12 & 4 \\
\hline Domain size & $270 \times 220$ & $265 \times 265$ & $310 \times 310$ \\
\hline Vertical levels & 36 & 36 & 36 \\
\hline Model top $(\mathrm{hPa})$ & 20 & - & - \\
\hline Time step (s) & 90 & 30 & 10 \\
\hline Cumulus convection & Tiedtke & Tiedtke & Explicit \\
\hline Boundary layer & Yonsei University (YSU) & - & - \\
\hline $\begin{array}{l}\text { Ocean mixed layer temperature } \\
\text { and depth }\end{array}$ & $\begin{array}{l}\text { Hybrid Coordinate Ocean Model } \\
\text { (HYCOM) analysis }\end{array}$ & - & - \\
\hline Ocean model & One-dimensional mixed layer & - & - \\
\hline Ocean-air flux & Donelan $C_{D}+$ Garratt $C_{K}$ & - & - \\
\hline Microphysics & WRF single-moment 6-class scheme (WSM6) & - & - \\
\hline Land surface & Noah land surface model (NLSM) & - & - \\
\hline Turbulence & Smagorinsky 2D & - & - \\
\hline Diffusion & Second-order diffusion & - & - \\
\hline Scalar advection & Positive definite & - & - \\
\hline $\begin{array}{l}\text { Radiation (longwave } \\
\text { and shortwave) }\end{array}$ & $\begin{array}{l}\text { Rapid Radiative Transfer Model } \\
\text { for GCMs (RRTMG) }\end{array}$ & - & - \\
\hline
\end{tabular}

Kuruppumullage Don et al. (2016) and Kowaleski and Evans (2016), using the method developed by Gaffney et al. (2007). We focus on results from track clustering throughout the paper; CPS path clustering is performed to help select the optimal track cluster partition. Although all WRF simulations end at 0000 UTC 1 November, track and CPS paths used in clustering are truncated at 0000 UTC 31 October to reduce the effect of postlandfall variation in Sandy's track and CPS evolution. Prior to clustering, CPS paths are smoothed by computing a 24-h running mean centered on each time step. All smoothed $B$ and $-\mathbf{V}_{L}^{T}$ values are then normalized as in Arnott et al. (2004).

In regression mixture-model clustering, each timedependent path (here, storm track or CPS path) is probabilistically assigned to models (clusters) by its fit to the central polynomial trajectory and error covariance matrix defining each model. Regression mixture-model clustering requires the selection of cluster shape, polynomial order, and number of clusters prior to clustering. All clusters are prescribed to have a circular error covariance matrix as in Kuruppumullage Don et al. (2016)
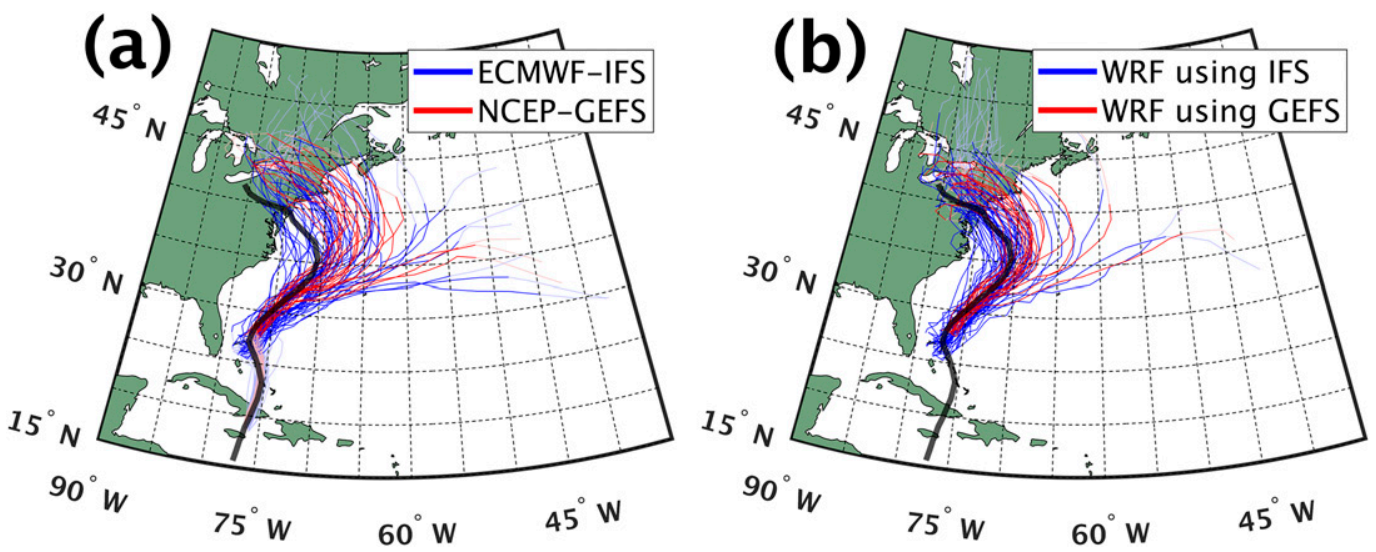

FIG. 6. Ensemble track forecasts of Hurricane Sandy from (a) ECMWF-IFS and NCEP-GEFS global models initialized at 0000 UTC 25 Oct, and (b) WRF simulations run using IFS and GEFS forecasts for initial and boundary condition. The dark portion of each track shows Sandy's path between 0000 UTC 27 Oct and 0000 UTC 31 Oct, the 96-h interval used in track and CPS clustering. The best track of Sandy in each panel is shown in black. 
and Kowaleski and Evans (2016). The optimal polynomial order and number of clusters are determined by comparing results produced by varying combinations of polynomial order (first-sixth order) and number of clusters (3-7).

For each combination of polynomial order and number of clusters (e.g., third-order trajectories with five clusters), cluster parameters and assignment probabilities are iterated through an expectation-maximization (EM) algorithm that converges on a maximum likelihood. Because the EM algorithm may converge on a local, rather than global, maximum likelihood (Gaffney et al. 2007), the clustering is repeated 500 times with random initial parameter values. The solution with the highest likelihood is chosen as the partition for that polynomial order and number of clusters combination. All candidate partitions are then compared to select the optimal polynomial order and number of clusters.

Kuruppumullage Don et al. (2016) and Kowaleski and Evans (2016) found that when clustering TC tracks or CPS paths, selecting the optimal polynomial order is more straightforward than selecting the optimal number of clusters. Bayesian information criterion (BIC) and mean squared displacement (MSD) are used to select the optimal polynomial orders for track and CPS cluster partitions. The BIC is computed from the log-likelihood, with larger penalties incurred when using more independent parameters (higher polynomial order and/or more clusters). It favors partitions that balance a high likelihood (good fit) with parsimony (lower polynomial order and fewer clusters; Kuruppumullage Don et al. 2016). The MSD is computed from the squared distance between the position (geographic or normalized CPS) of each ensemble member and that of its cluster polynomial trajectory at each time; in calculating MSD, ensemble members are assigned based on their highest probability of cluster membership. MSD heavily penalizes partitions with outlier members; however, it does not penalize partitions with a higher polynomial order and/or more clusters. For both BIC and MSD, lower values indicate greater support for the partition being evaluated.

As expected, BIC favors lower polynomial orders than MSD for track and CPS clustering (Fig. 7). For track partitions employing 3-7 clusters, BIC values begin to increase (negative decrease) beyond fourth order (Fig. 7a), while MSD continues to decrease through sixth order (Fig. 7b). However, the decrease in MSD drops off substantially between fourth and fifth order. For CPS clustering, BIC begins to increase between fifth and sixth order (Fig. 7c), and MSD decrease is minimal (Fig. 7d). Therefore, fourth- (fifth) order polynomials are selected for track (CPS) clustering.
After selecting optimal polynomial orders for track and CPS clustering, the optimal number of clusters must be chosen. Neither BIC nor MSD provides a clear indication of the optimal number, so we turn to another mode of analysis. Because we seek to relate variations in the track and structural evolution of Hurricane Sandy, we examine the similarity between the track- and CPSbased partitions. The Rand index (RI; Rand 1971) and adjusted Rand index (ARI; Hubert and Arabie 1985) are employed to examine this membership correspondence. The RI, which varies between 0 and 1 , describes the similarity between two clustering partitions; the ARI, which varies between -1 and 1 , also describes this similarity, but corrects for the agreement expected from chance. An ARI value above 0 indicates that two cluster partitions are more similar than would be expected from chance.

RI and ARI values are computed for each combination of fourth-order track and fifth-order CPS partitions with three through seven clusters (Fig. 8). Based on both RI and ARI, the highest membership correspondence is found for partitions in which both track and CPS have either six or seven clusters. Because we seek partitions that yield strong track-structure correspondence with no more clusters than necessary, six-cluster solutions are selected for both the track and CPS partitions.

\section{Results}

\section{a. Cluster-mean track and CPS characteristics}

The chosen mixture model specifications for track clustering yield a coherent partition among geographic tracks in the 72-member WRF ensemble (Fig. 9a); clustering using the CPS specifications also results in a coherent partition among CPS paths (Fig. 9c). Substantial similarities exist between the cluster-mean tracks produced by geographic track clustering (Fig. 9a) and the cluster-mean tracks produced by CPS clustering (Fig. 9b; Table 2). Substantial agreement also exists between the cluster-mean CPS paths produced by CPS clustering (Fig. 9d) and by track clustering (Fig. 9c). The positive ARI values across all combinations of track and CPS cluster numbers (Fig. 8b) indicate that this track-CPS correspondence is a robust feature of the WRF ensemble.

Among track clusters (Fig. 9a), members of the westernmost cluster (red; 3 members) landfall the earliest and farthest south; mean landfall occurs at 0115 UTC 29 October and the cluster-mean track landfalls on the Delmarva Peninsula. Moving from west to east, the magenta cluster (19 members) and blue cluster (27 members) straddle Sandy's observed best 

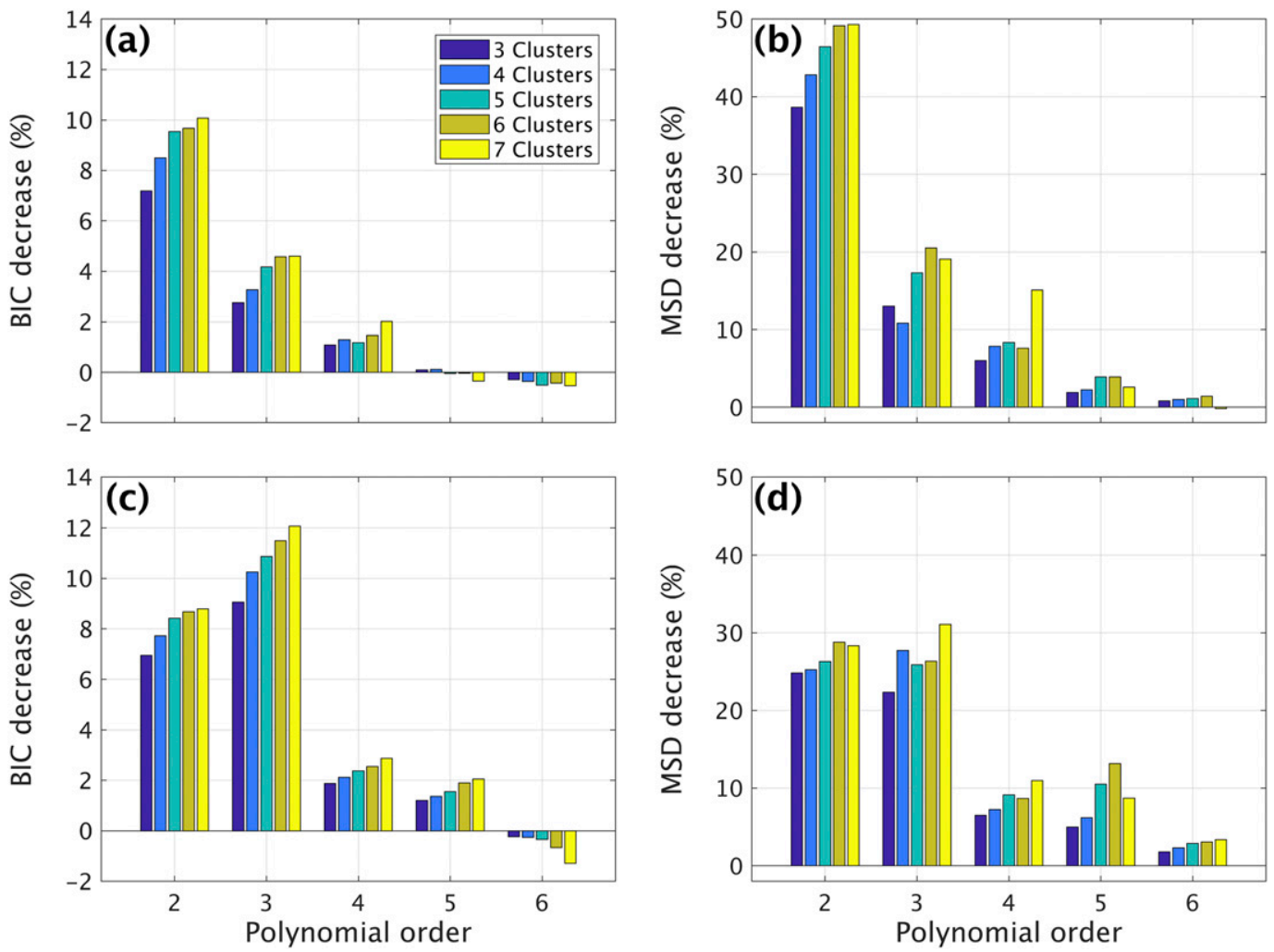

FIG. 7. Percentage decrease with each additional polynomial order for track clustering using (a) Bayesian information criterion (BIC) and (b) mean squared displacement (MSD); percentage decrease with each additional polynomial order for CPS path using (c) BIC and (d) MSD. In all cases, partitions employing three-seven clusters are being evaluated.

track, with the magenta (blue) mean path slightly to the west (east) of the observed track. Magenta (blue) cluster members have a mean landfall at 1845 UTC 29 October (0700 UTC 30 October) in southern (northern) New Jersey. In the cyan cluster (13 members), Sandy tracks substantially east of the blue cluster after 28 October, making a mean landfall in southeast New England at 1230 UTC 30 October. Members of the green cluster (7 members) move the farthest eastward before turning northward and northwestward. Green cluster members
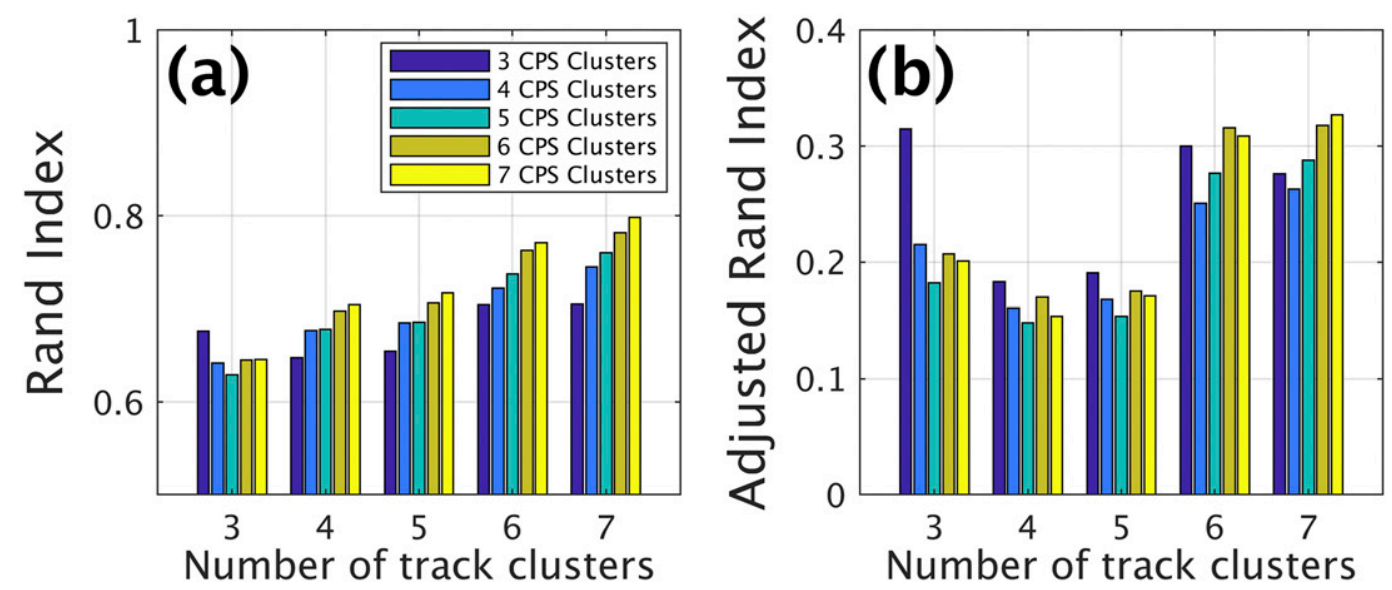

FIG. 8. Similarity of cluster membership between fourth-order track cluster partitions and fifth-order CPS cluster partitions, each using three-seven clusters, based on the (a) Rand index and (b) adjusted Rand index. 

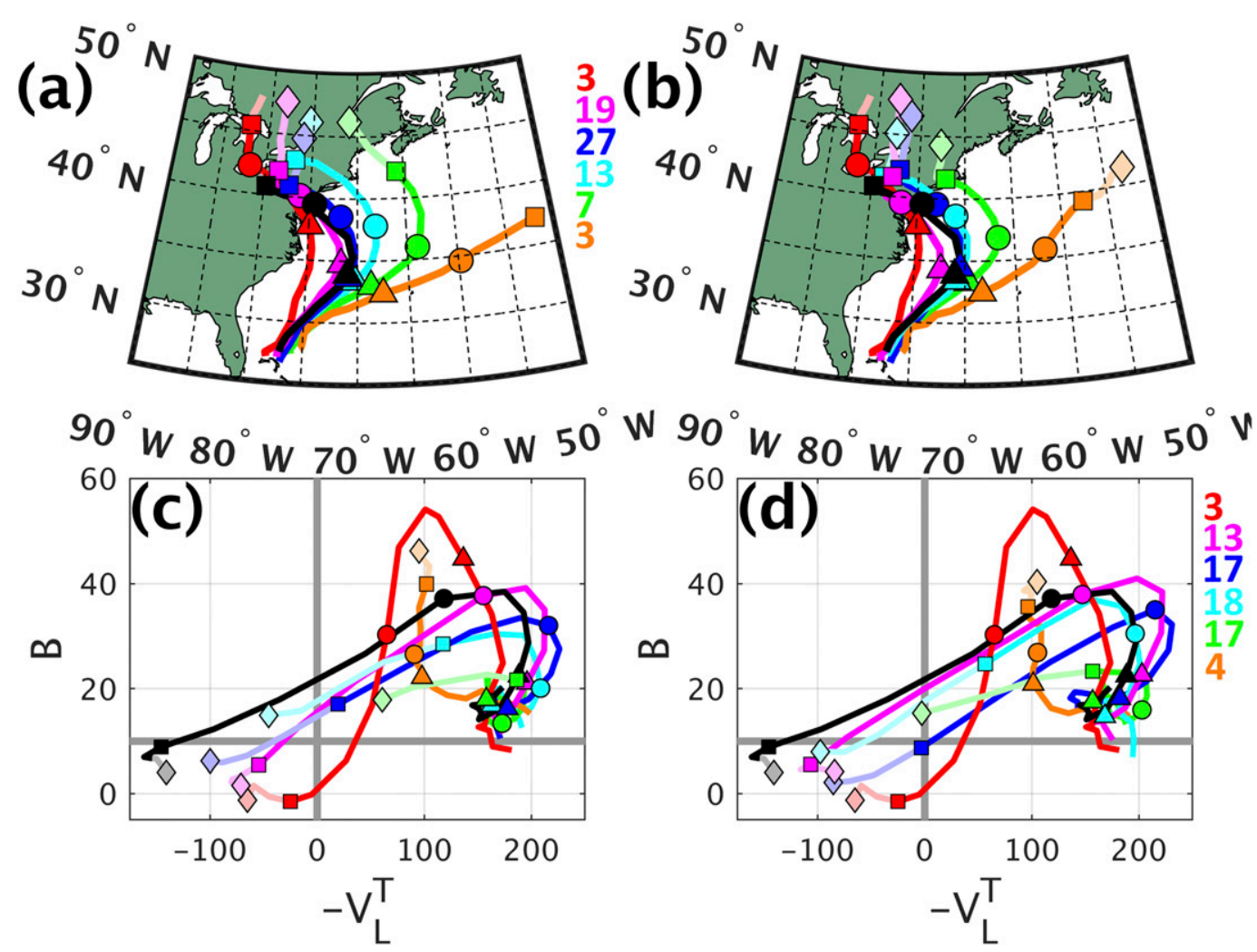

FIG. 9. For track and CPS cluster partitions: (a) mean track per track cluster, (b) mean track per CPS cluster, (c) mean CPS path per track cluster, and (d) mean CPS path per CPS cluster. Track and CPS cluster populations are indicated to the right of (a) and (d), respectively. Triangles, circles, squares, and diamonds indicate the position/ structure of Sandy at 0000 UTC 29, 30,31 Oct, and 1 Nov, respectively. In (a) and (b) the best track is represented in black. In (c) and (d) the CPS path of Sandy from a WRF simulation using ECMWF analysis conditions (ECMWF 2011) is represented in black. The faded colors at the end of each track and CPS path represent the track of Sandy after 0000 UTC 31 Oct, which was not included in clustering.

make a mean landfall in Maine at 0745 UTC 31 October. Simulations in the orange cluster ( 3 members) move eastward, out to sea.

Although the positive ARI (0.316) demonstrates that track and CPS evolution are related, the three most populous track clusters (blue, magenta, and cyan) undergo qualitatively similar cluster-mean CPS evolutions (Fig. 9c). Each cluster experiences a rapid increase in storm asymmetry $(B)$, followed by a rapid decrease in
$-\mathbf{V}_{L}^{T}$ near and after landfall (cf. Figs. 9a and 9c). This path indicates warm seclusion formation as cool, midlatitude air first encroaches on the west and south sides of Sandy (increasing $B$ ), while the lower-tropospheric warm core remains intact (highly positive $-\mathbf{V}_{L}^{T}$ ). Later, cool air reaches the center of Sandy and $-\mathbf{V}_{L}^{T}$ drops rapidly, eventually becoming negative. This evolution is similar to Sandy's CPS evolution diagnosed from a hindcast WRF simulation that uses ECMWF analysis

TABLE 2. Track and CPS membership intercomparision for the clustering partitions employed in this study. Mean tracks and CPS paths of each cluster are shown in Fig. 9.

\begin{tabular}{|c|c|c|c|c|c|c|c|}
\hline & CPS red & CPS magenta & CPS blue & CPS cyan & CPS green & CPS orange & Total \\
\hline Track red & 3 & 0 & 0 & 0 & 0 & 0 & 3 \\
\hline Track magenta & 0 & 12 & 4 & 3 & 0 & 0 & 19 \\
\hline Track blue & 0 & 1 & 12 & 12 & 2 & 0 & 27 \\
\hline Track cyan & 0 & 0 & 1 & 3 & 8 & 1 & 13 \\
\hline Track green & 0 & 0 & 0 & 0 & 7 & 0 & 7 \\
\hline Track orange & 0 & 0 & 0 & 0 & 0 & 3 & 3 \\
\hline Total & 3 & 13 & 17 & 18 & 17 & 4 & $n=72$ \\
\hline
\end{tabular}


data for initial and boundary conditions (black path in Figs. 9c and 9d), and the CPS evolution calculated directly from the ECMWF analysis (ECMWF 2011; Fig. 1).

The mean CPS path of the green cluster exhibits a smaller $B$ increase than the magenta, blue, and cyan paths, though the rapid $-\mathbf{V}_{L}^{T}$ decrease as the warm core decays is comparable to that observed in the other three clusters. The two small outlier clusters (red and orange; three members each) undergo substantially different CPS evolutions from the larger clusters. The red cluster experiences a rapid asymmetry increase and subsequent decrease, while the orange cluster retains a lowertropospheric warm core as it becomes increasingly asymmetric between 29 October and 1 November. For the rest of the paper, we will focus on results from the four most populous clusters (blue, magenta, cyan, and green), which comprise 66 members (92\%) of the WRF ensemble.

\section{b. Timing of Sandy-trough interaction and extratropical transition}

Though Sandy interacts with a midlatitude trough and undergoes ET in each of the four most populous clusters, the clusters manifest clear differences in the timing of the Sandy-trough interaction, as shown by intercluster variations in the evolution of the cluster-mean ${ }^{2}$ azimuthally averaged upper-level eddy momentum flux convergence (EMFC; Figs. 10a-d). The increase in mean $200-300-\mathrm{hPa}$ eddy momentum forcing from the trough at radii $>500 \mathrm{~km}$ occurs earliest in the magenta cluster; more eastward clusters show a later increase. For example, mean 200-300-hPa EMFC averaged in the 500-1000-km annulus around Sandy's center first exceeds $50 \mathrm{~m} \mathrm{~s}^{-1}$ day $^{-1}$ at 0600 UTC 29 October in the magenta composite (Fig. 10e), compared to 2100 UTC 29 October in blue (Fig. 10f) and 0900 UTC 30 October in cyan (Fig. 10g). In the green composite, mean 200-300-hPa EMFC in the 500-1000-km annulus never exceeds $40 \mathrm{~m} \mathrm{~s}^{-1}$ day $^{-1}$ during the Sandymidlatitude trough interaction, indicating weaker maximum upper-level forcing in that cluster (cf. Figs. 10h and $10 \mathrm{e}-\mathrm{g})$.

In addition to the trough's momentum forcing on Hurricane Sandy, the Sandy-trough interaction can be described by the impingement of upper-level negative PV advection from Sandy's outflow on the trough. Comparison of the cluster-mean 250-hPa PV advection by the

\footnotetext{
${ }^{2}$ To account for variations in track among cluster members and to preserve features of the evolution common to the members, all cluster means depicted here are storm relative.

${ }^{3} \mathrm{PV}$ advection by the divergent wind was not computed because of the regional domain of the WRF simulations.
}

total wind ${ }^{3}$ in the northwest quadrant of Sandy reveals intercluster variations in the timing and magnitude of the interaction between Sandy's outflow and the midlatitude trough (Fig. 11). Progressively farther east clusters have later and weaker maximum values of negative PV advection northwest of Sandy averaged in the 1000-2000$\mathrm{km}$ annulus (Figs. 11e-h). Thus, the magenta and blue clusters show earlier and more intense upper-level trough interaction, especially relative to the green cluster.

Earlier storm-trough interaction in the more westward clusters results in earlier warm seclusion formation and ET completion (Fig. 12). Seclusion formation and contraction are evident in the mean $850-\mathrm{hPa} \theta_{e}$ of the magenta cluster between 0600 UTC 29 October (Fig. 12a) and 1800 UTC 29 October (Fig. 12e); inward movement of the 700-hPa frontogenesis axis accompanies the contraction (Figs. 12a,e). By 0600 UTC 30 October, the warm seclusion in the magenta cluster has eroded, as low $-\theta_{e}$ air from the trough has reached Sandy's center at low levels (Fig. 12i).

In the blue cluster composite, a warm seclusion forms by 1800 UTC 29 October (Fig. 12f), while the seclusion and frontogenesis region contract toward the center of Sandy by 0600 UTC 30 October (Fig. 12j). The beginning of warm seclusion formation is evident in the cyan composite at 0600 UTC 30 October (Fig. 12k); by 1800 UTC 30 October (Fig. 12o) the seclusion has contracted and decayed substantially. The easternmost green composite, in which Sandy interacts with the trough latest, shows only the early stages of warm seclusion formation at 1800 UTC 30 October (Fig. 12p).

Analysis of intercluster variation of PV on the 325-K isentrope during the cyclonic wave break and wrap-up of Sandy by the trough (Fig. 13) complements the discussion of earlier warm seclusion formation and decay in more westward simulations. This isentropic level captures the descent of PV from the midlatitude trough and wrap-up of Sandy at small radii. The PV wave breaking and wrap-up of Sandy occur earliest in the magenta cluster and progressively later in more eastward clusters. At 1800 UTC 29 October, the magenta cluster composite shows PV values above 2 PVU (the dynamic tropopause value; Hoskins and Berriford 1988; Hoskins 1997) wrapping around the south side of Sandy less than $400 \mathrm{~km}$ from the TC center (Fig. 13e). In the blue and cyan composites, these high PV values remain well west of Sandy (Figs. 13f,g), while the green composite shows the least connection between the PV signatures of the trough and Sandy.

Twelve hours later (0600 UTC 30 October), the 325-K PV cyclonic wave break has advanced greatly in the magenta composite; PV values exceeding 2 PVU (1 PVU $=10^{-6} \mathrm{~K} \mathrm{~kg}^{-1} \mathrm{~m}^{2} \mathrm{~s}^{-1}$ ) have wrapped around 

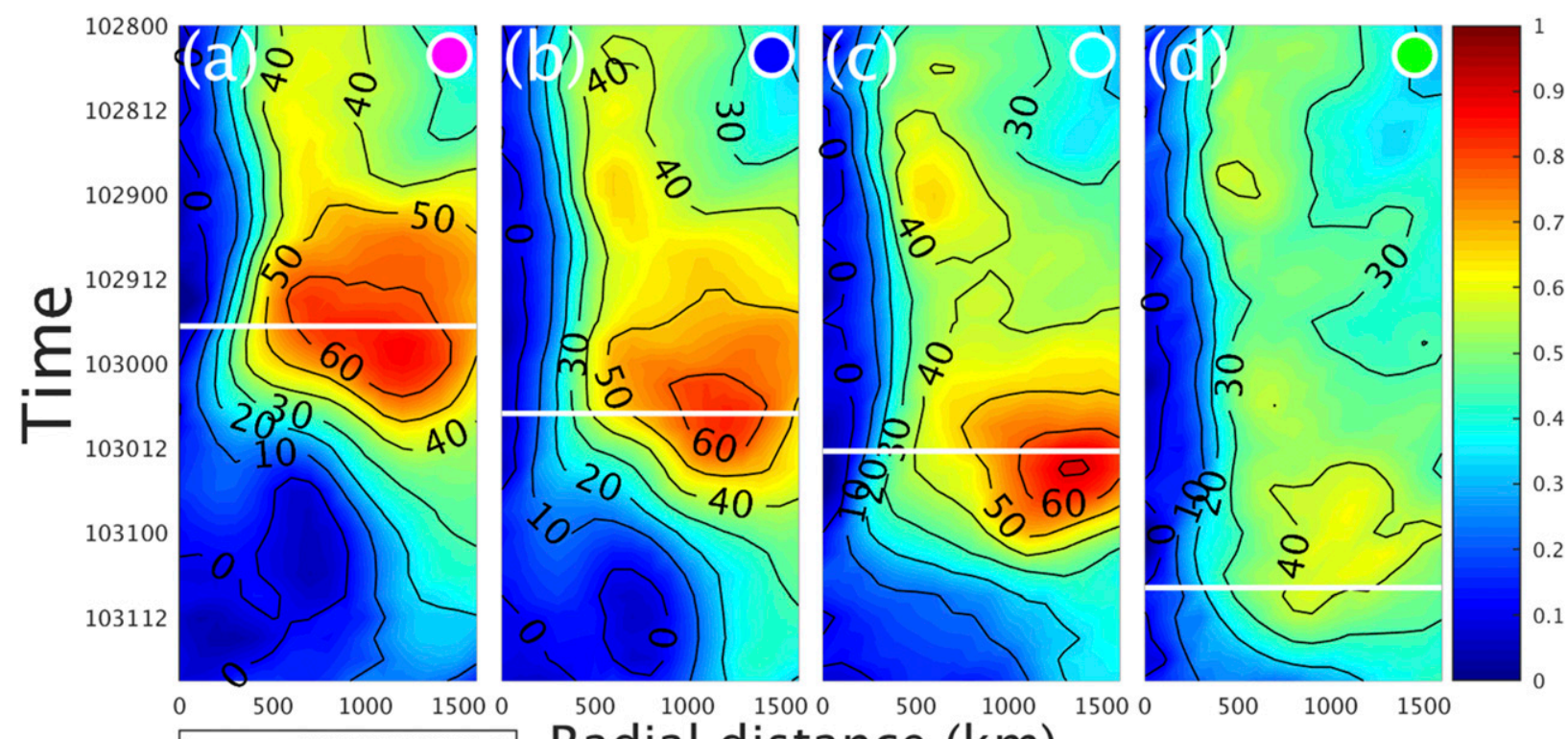

RMFC (500-1000 km) Radial distance $(\mathrm{km})$
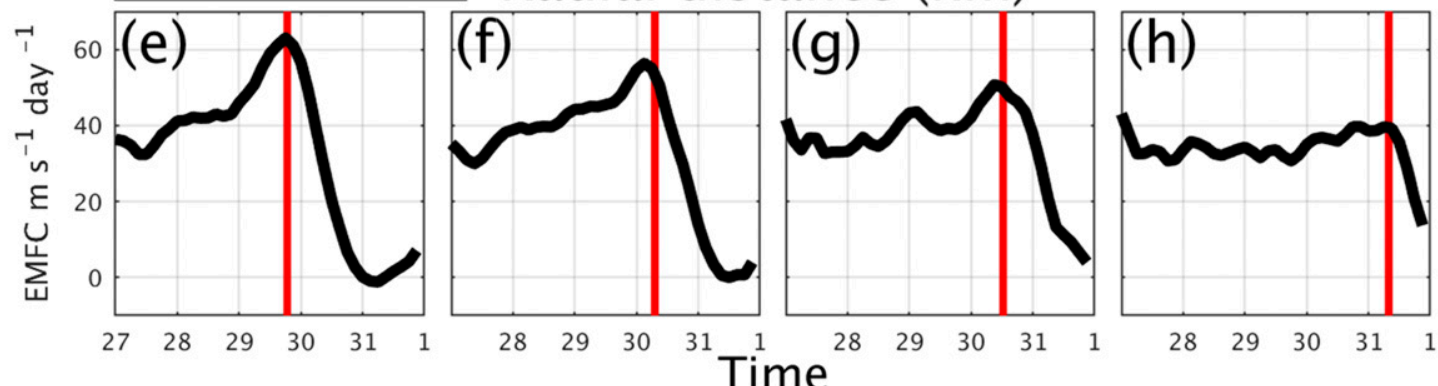

FIG. 10. (a)-(d) Cluster-mean azimuthally averaged eddy momentum flux convergence (EMFC) averaged from 200-300 hPa in the magenta, blue, cyan, and green clusters and (e)-(h) time evolution of EMFC averaged from 200-300 hPa and 500-1000 km. The mean landfall time of each cluster is indicated by a white line in (a)-(d) and a red line in (e)-(h). Data in this figure and Figs. 11 and 18 are calculated from the outermost WRF domain (36-km resolution). This figure and Figs. 11-20 omit data from the WRF simulation derived from IFS member 4 (blue cluster), because Sandy did not make landfall in that simulation.

the east side of Sandy; concurrently, the 250-hPa jet streak south of Sandy has acquired a more southwestnortheast orientation, and a large region of winds greater than $25 \mathrm{~m} \mathrm{~s}^{-1}$ exists east of Sandy (Fig. 13i). In the blue composite (Fig. 13j), PV values exceeding 2 PVU have advanced along the south side of Sandy; the wave breaking progresses substantially during the next $12 \mathrm{~h}$ (Fig. 13n). Wave breaking in the cyan composite is under way by 1800 UTC 30 October (Fig. 13o), while in the green composite the Sandy-trough interaction remains the least advanced; the 2-PVU contour of the trough remains well southwest of Sandy (Fig. 13p).

Evolution of $900-\mathrm{hPa} \mathrm{PV}$ (thick black contours in Fig. 13) among the four clusters also reflects intercluster timing differences in Sandy-trough interaction and the warm seclusion evolution. Galarneau et al. (2013) showed that as the warm seclusion formed and contracted, a strip of lower-tropospheric PV west of Sandy's center was wrapped cyclonically into the cyclone's inner core (their Fig. 9). Signals of this inner-core PV concentration are found across the magenta, blue, and cyan composites in Fig. 13. In the magenta composite, a streamer of elevated 900-hPa PV extends west and southwest of Sandy's center at 0600 UTC 29 October (Fig. 13a). By 1800 UTC, the PV has concentrated in Sandy's center and the $900-\mathrm{hPa}$ PV field has acquired a more circular appearance (Fig. 13e). In more eastward clusters, low-level PV concentration occurs later; in the blue composite it occurs between 1800 UTC 29 October (Fig. 13f) and 0600 UTC 30 October (Fig. 13j), while in the cyan composite it is completed after 0600 UTC 30 October (Fig. 13k). In the green composite, low-level PV concentration has not been completed through 1800 UTC 30 October (Fig. 13p), a result of later Sandy-trough interaction.

Timing differences in warm seclusion evolution as reflected by lower-tropospheric $\theta_{e}$ and PV among the four 


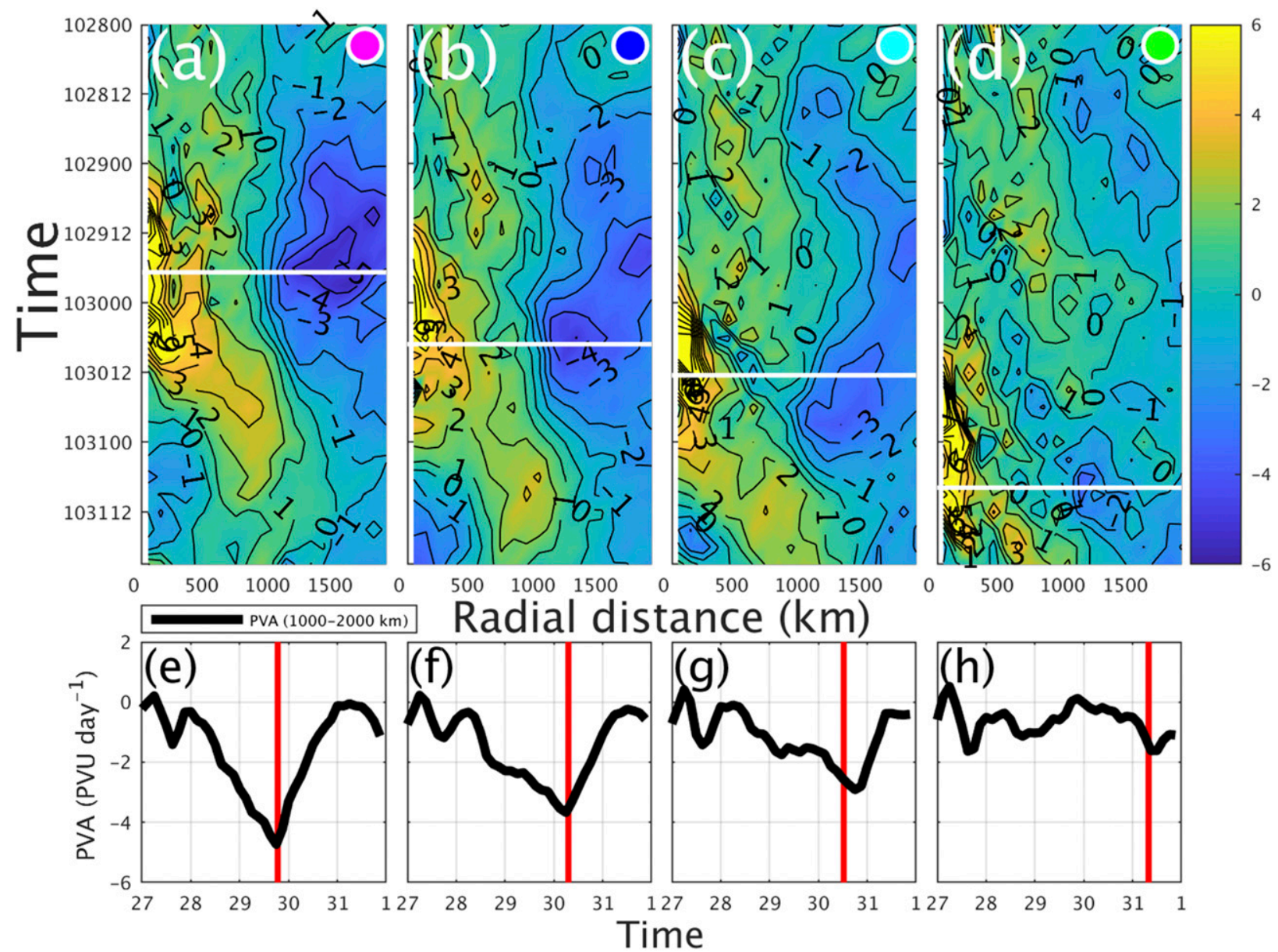

FIG. 11. (a)-(d) Cluster-mean 250-hPa potential vorticity advection (PVA) in the northwest quadrant relative to Sandy's center and (e)-(h) time evolution of 250-hPa PVA in the northwest quadrant averaged from 1000 to $2000 \mathrm{~km}$ from Sandy's center. The mean landfall time of each cluster is indicated by a white line in (a)-(d) and a red line in (e)-(h).

clusters (Fig. 14) corroborate differences between the synoptic composites. As cool lower-tropospheric air from the trough sweeps around Sandy, while high $\theta_{e}$ values persist at small radii, the radial $\theta_{e}$ gradient increases (Fig. 14a). During warm seclusion contraction, low-level PV is concentrated in Sandy's core (Fig. 12), as demonstrated by the rapid increase in mean $0-50-\mathrm{km} 900-\mathrm{hPa}$ PV observed in each cluster (Fig. 14b). The increase in the radial $850-\mathrm{hPa} \theta_{e}$ gradient and in $900-\mathrm{hPa} \mathrm{PV}$ at small radii occurs progressively later in more eastward clusters. The radial $850-\mathrm{hPa} \theta_{e}$ gradient also collapses later in more eastward clusters, as cool lower-tropospheric air reaches Sandy's inner-core later among these simulations.

\section{c. Landfall-relative differences in extratropical transition}

The four analyzed clusters exhibit clear differences in the timing of the Sandy-trough interaction and warm seclusion formation; however, these differences decrease substantially when examining the structural evolution of Sandy relative to the storm's landfall time in each WRF simulation. In each cluster composite, a warm seclusion forms during the $24 \mathrm{~h}$ before landfall as the trough captures Sandy and low- $\theta_{e}$ air rotates cyclonically around the storm at low levels. During this process, low-level radial baroclinicity increases, especially southwest of Sandy's center (cf. Figs. 15i-1 and $15 \mathrm{a}-\mathrm{d})$. This increase in low-level baroclinicity intensifies both the primary and secondary circulation of Sandy, as diagnosed by the Sawyer-Eliassen balanced vortex response of Galarneau et al. (2013). During the final $12 \mathrm{~h}$ before landfall, the warm seclusion contracts in each composite (cf. Figs. $15 \mathrm{~m}-\mathrm{p}$ and $15 \mathrm{e}-\mathrm{h}$ ). Radially inward movement of $700-\mathrm{hPa}$ frontogenesis (white contours in Fig. 15) accompanies the seclusion contraction in each cluster, consistent with the balanced vortex response. At landfall, the four cluster composites have a similar large-scale 

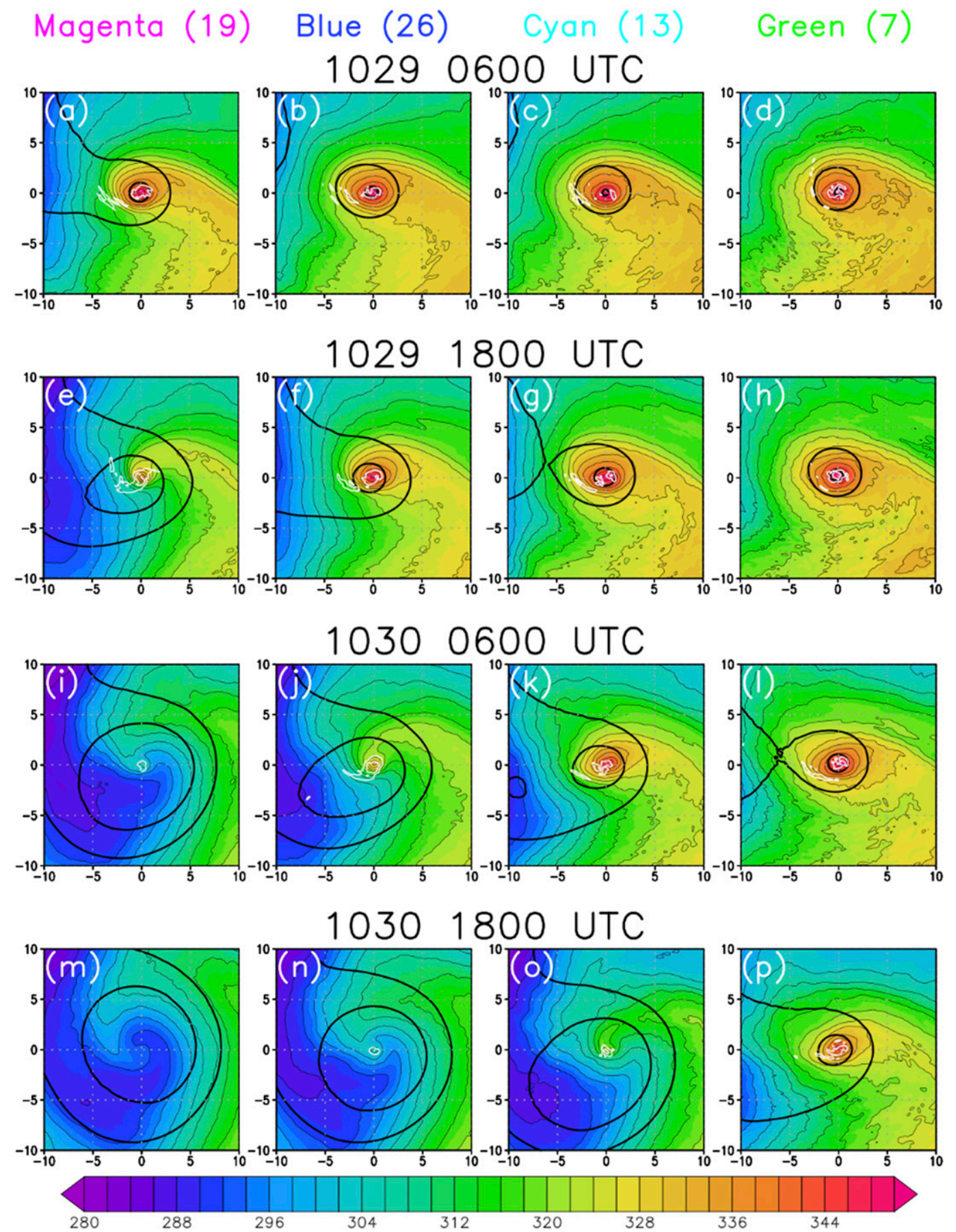

FIG. 12. For the magenta, blue, cyan, and green clusters: composites of 850 -hPa equivalent potential temperature (K; shaded and thin black contours every $4 \mathrm{~K}$ ), 500-hPa geopotential height (thick black contours at 5440 and $5560 \mathrm{~m}$ ), and $700-\mathrm{hPa}$ frontogenesis (white contours at 1 and $2 \mathrm{~K} \mathrm{~m}^{-1} \mathrm{~s}^{-1} \times 10^{7}$ ) at (a)-(d) 0600 UTC 29 Oct, (e)-(h) 1800 UTC 29 Oct, (i)-(l) 0600 UTC 30 Oct, and (m)-(p) 1800 UTC 30 Oct. Numbers at the top indicate the number of members in each composite. Data in this figure and Figs. 13, 15-16, and 19-20 are generated from the intermediate WRF domain (12-km resolution). 

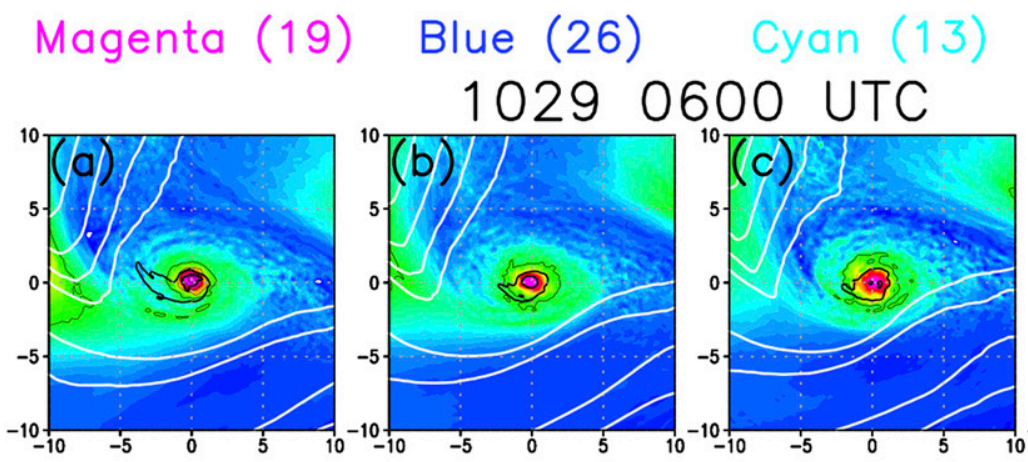

Green
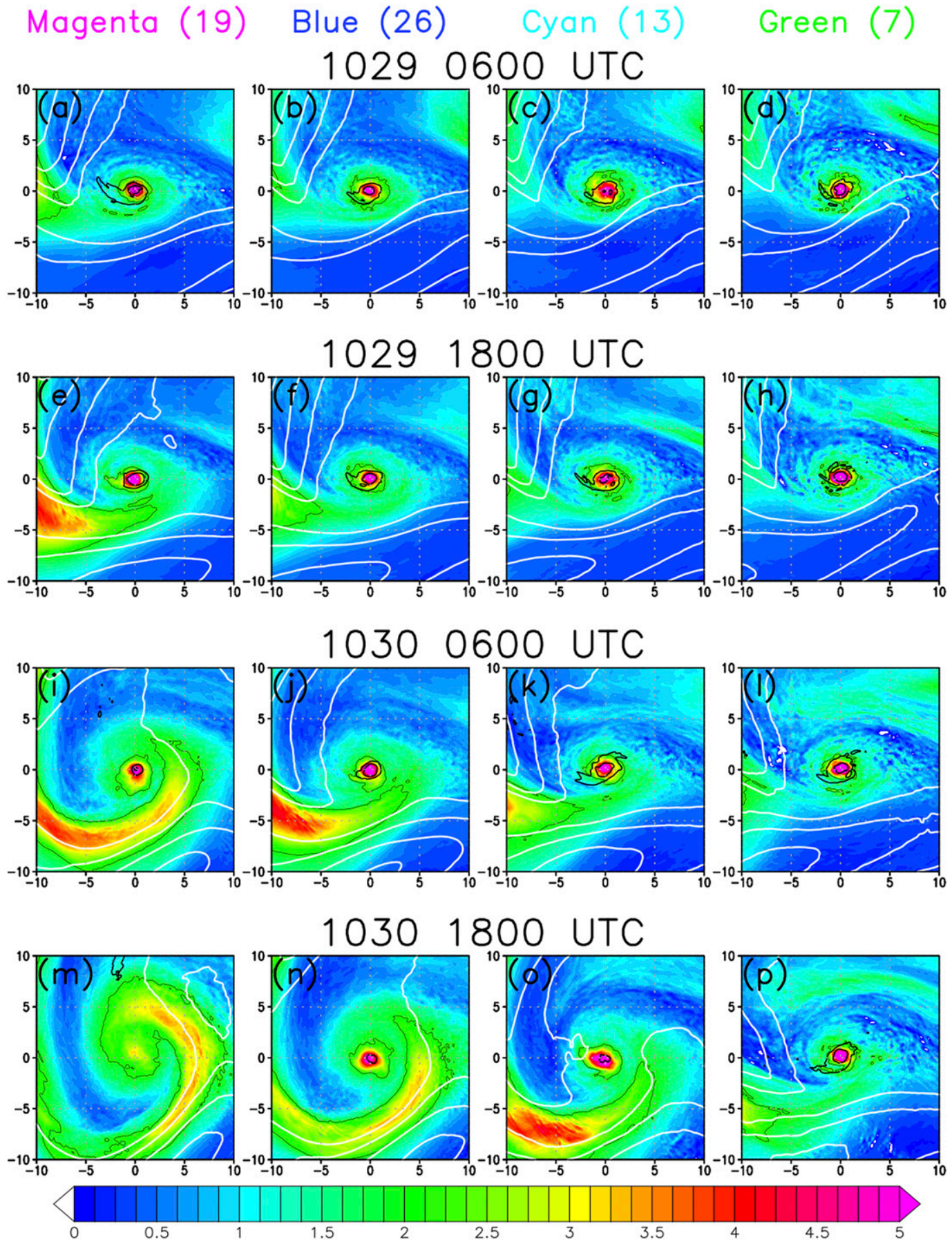

FIG. 13. Cluster composites of potential vorticity on the 325-K isentrope (PVU; shaded and thin black contour at 2 PVU), 900-hPa potential vorticity (thick black contours at 2 and 4 PVU), and 250-hPa wind speed (white contours every $10 \mathrm{~m} \mathrm{~s}^{-1}$ between 25 and $55 \mathrm{~m} \mathrm{~s}^{-1}$ ) at (a)-(d) 0600 UTC 29 Oct, (e)-(h) 1800 UTC 29 Oct, (i)-(l) 0600 UTC 30 Oct, and (m)-(p) 1800 UTC 30 Oct.

thermodynamic structure: a small contracting seclusion of high 850-hPa $\theta_{e}$ values near Sandy's center, with much lower $\theta_{e}$ values wrapping around the south and southeast sides of the storm.
Intercluster similarities in the large-scale structural evolution of Sandy near landfall are also evident from the landfall-relative PV evolution (Fig. 16). In each composite, high-PV air from the trough moves to the 


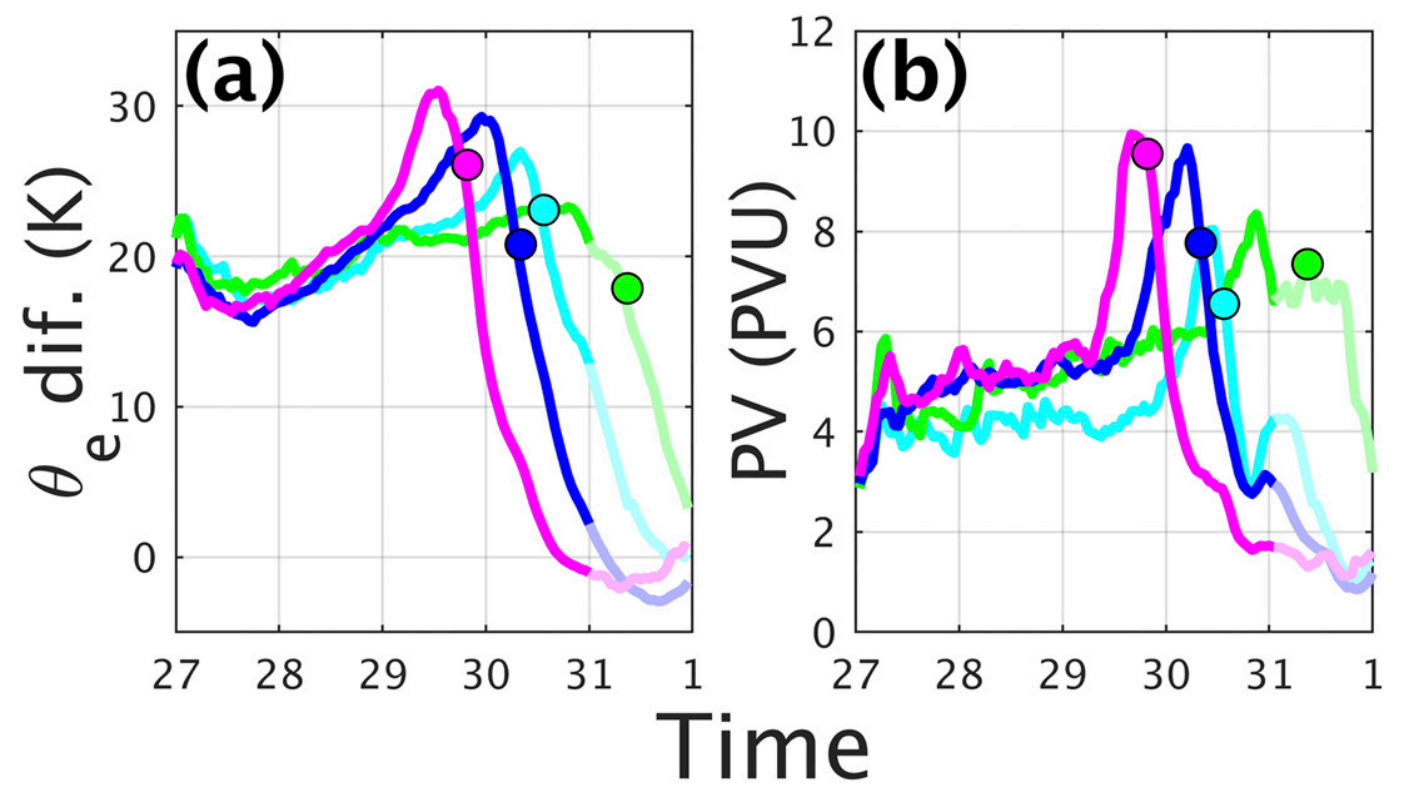

FIG. 14. Cluster-mean time evolution of (a) mean 850 -hPa equivalent potential temperature difference between 0-50 and 200-400 km from the center of Sandy and (b) mean 900-hPa potential vorticity $0-50 \mathrm{~km}$ from the center of Sandy. The mean landfall time of Sandy in each cluster is indicated by a colored circle. Data in this figure and Fig. 17 are generated from the innermost WRF domain (4-km resolution).

south of Sandy and wraps around the storm at small radii during the $24 \mathrm{~h}$ prior to landfall. At the time of landfall, each composite shows a strip of 325-K PV $>2$ PVU south of Sandy, with lower values wrapping around Sandy's core, and the highest values well southwest of the storm (Figs. 16m-p). This PV wrap-up, and the associated increase in low-level baroclinicity (Fig. 15) and convergence, accompany the concentration of $900-\mathrm{hPa}$ PV (thick black contours in Fig. 16) generated along the frontogenesis axis (Fig. 15) into Sandy's inner core (cf. Figs. $16 \mathrm{~m}-\mathrm{p}$ and $16 \mathrm{e}-\mathrm{h}$ ). The marked increase in 900-hPa PV at $0-50-\mathrm{km}$ radii and decrease at 100 $200-\mathrm{km}$ radii shortly before landfall is evident across the four clusters (Figs. 17a,b). This low-level PV concentration at small radii near landfall is robust across the populations of the four clusters; at least $80 \%$ of the members of each cluster attain their highest $0-50-\mathrm{km}$ 900-hPa PV within five hours of landfall.

Despite comparable large-scale structural evolutions among the four clusters, subtle, but relevant, differences exist, particularly between the green cluster and the three more westward clusters. The green cluster composite shows greater warm seclusion erosion before landfall than the other clusters (cf. Figs. 15p and 15m-o). At landfall, green cluster members have a mean $0-50-\mathrm{km}$ $850-\mathrm{hPa} \theta_{e}$ of $330 \mathrm{~K}$, substantially lower than the other three clusters (337.4-344.2 K), and statistically significantly lower ( $95 \%$ confidence; two-tailed $t$ test) than cyan and magenta. The green composite also has the weakest radial $\theta_{e}$ gradient before and at landfall (Fig. 15), consistent with its lower $B$ values $(900-600-\mathrm{hPa}$ thickness asymmetry) during the warm seclusion (Fig. 9c). This weaker baroclinicity is also demonstrated by clustermean plots of the average $850-\mathrm{hPa} \theta_{e}$ difference between 0-50- and 200-400-km radii (Fig. 17c). Green has the lowest $\theta_{e}$ difference throughout the warm seclusion, including at landfall. Thus, green cluster members have the least intense warm seclusion (as measured by the radial gradient of $850-\mathrm{hPa} \theta_{e}$ ) and the greatest decay of inner-core high- $\theta_{e}$ values by landfall.

The weaker radial $\theta_{e}$ gradient in the green cluster occurs in conjunction with a less intense interaction between Sandy and the midlatitude trough, as demonstrated by cluster composites of landfall-relative $200-300-\mathrm{hPa}$ EMFC (cf. Figs. 18d and 18a-c). Cluster-composite plots of 500-hPa PV (Figs. 19a-d) at landfall also show the comparatively weak Sandy-trough interaction in the green cluster. In the green composite (Fig. 19d), the $\mathrm{PV}$ of Sandy at landfall remains more distinct from that of the trough at $500 \mathrm{hPa}$ than in the other three composites (Figs. 19a-c). Therefore, in the green cluster, the PV of the trough wrapping around Sandy does not extend as low in the troposphere as in the other three clusters.

Based on the Sawyer-Eliassen vortex balance solution that Galarneau et al. (2013) diagnosed in Sandy, a weaker increase in lower-tropospheric baroclinicity (weaker frontogenesis) yields weaker low-level convergence 

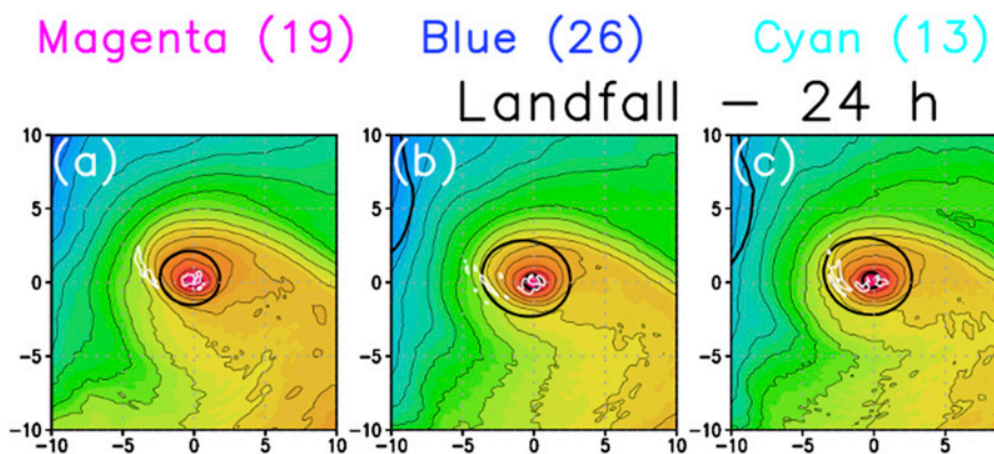

Green

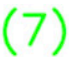

\section{Landfall - $24 \mathrm{~h}$}
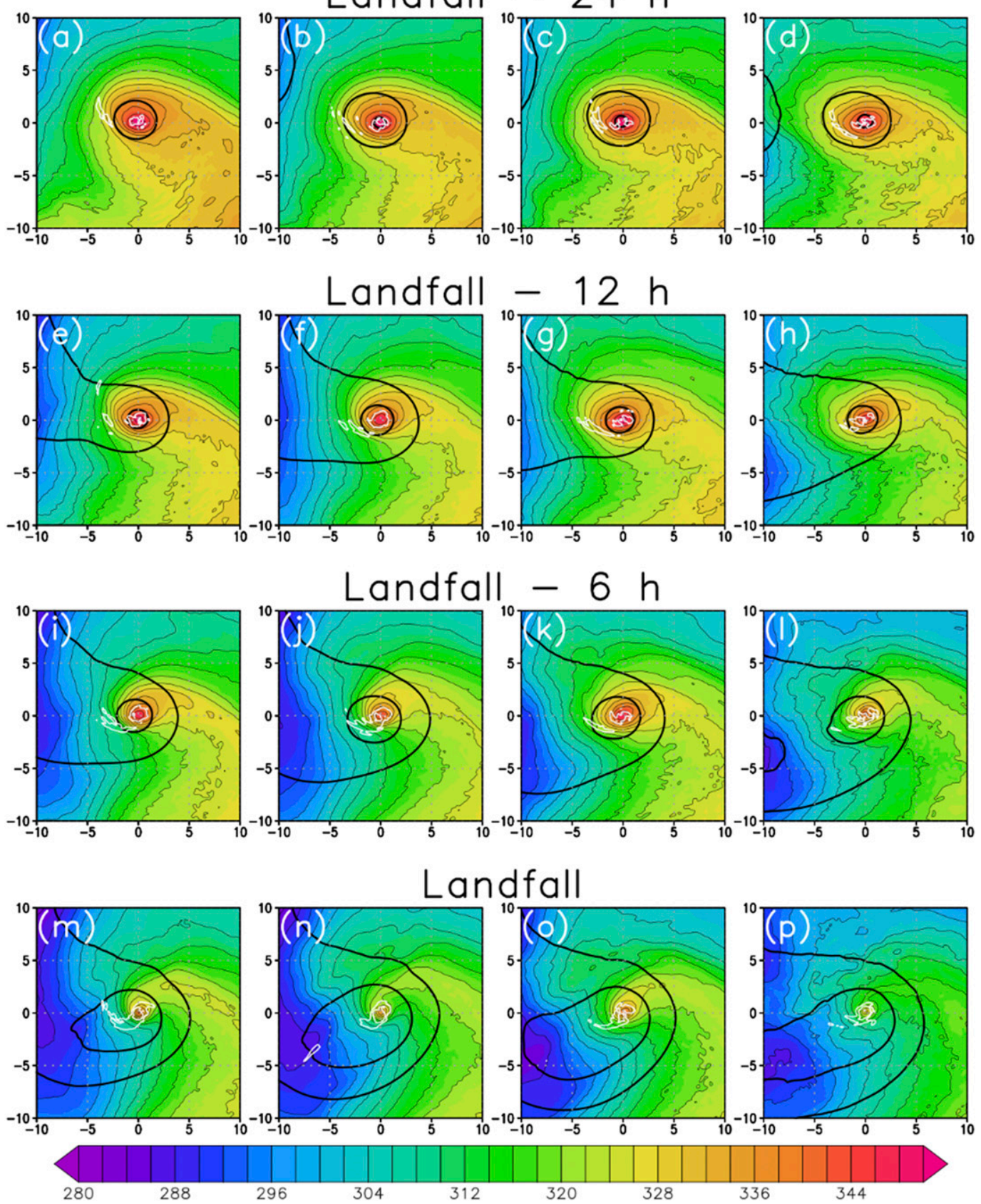

FIG. 15. As in Fig. 12, but composites are generated (a)-(d) $24 \mathrm{~h}$ before landfall, (e)-(h) $12 \mathrm{~h}$ before landfall, (i)-(l) $6 \mathrm{~h}$ before landfall, and (m)-(p) at the landfall time of each WRF ensemble member. The landfall time of each simulation in this figure and in Figs. 16-17 and Figs. 19-20 is approximated as the last hourly model output time before landfall.

and a slower contraction of the warm seclusion. Indeed, the green cluster has substantially weaker radial inflow at $850 \mathrm{hPa}$ than other clusters during the $12 \mathrm{~h}$ before landfall (cf. Figs. $18 \mathrm{~h}$ and $18 \mathrm{e}-\mathrm{g}$ ). Thus, the greater decay of the warm seclusion in the green cluster occurs despite weaker vortex contraction.

Green cluster members encounter lower SSTs before landfall (solid lines in Fig. 17e), which contribute to 

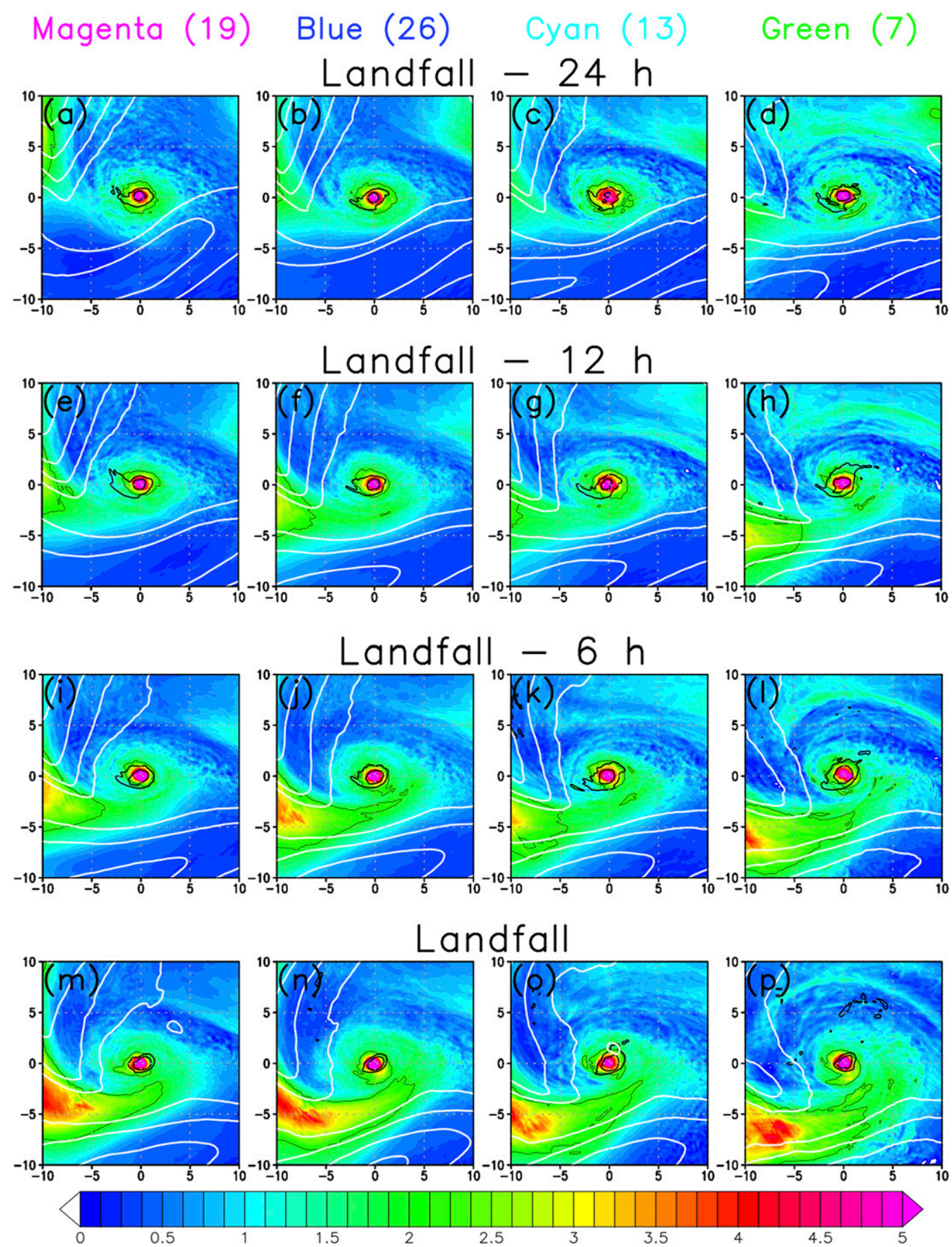

FIG. 16. As in Fig. 13, but composites are generated (a)-(d) $24 \mathrm{~h}$ before landfall, (e)-(h) $12 \mathrm{~h}$ before landfall, (i)(l) $6 \mathrm{~h}$ before landfall, and (m)-(p) at the landfall time of each WRF ensemble member.

greater warm core decay by landfall. During the $24 \mathrm{~h}$ before landfall, green members experience a mean SST of $19.5^{\circ} \mathrm{C}$ within a $250-\mathrm{km}$ radius, compared to $22.6^{\circ}-23.4^{\circ} \mathrm{C}$ in the other three clusters. Lower SSTs contribute to a lower mean 0-250-km enthalpy flux over water: $207 \mathrm{~W} \mathrm{~m}^{-2}$ during the $24 \mathrm{~h}$ before landfall, versus
$376-493 \mathrm{~W} \mathrm{~m}^{-2}$ in the more westward clusters (dashed lines in Fig. 17e). This reduced ocean-air energy transfer allows the lower troposphere to cool more rapidly. It also suppresses convection, as shown by the green cluster's lower mean 850-200-hPa integrated vertical water vapor flux inside $250 \mathrm{~km}$ (Fig. 17d). Less latent 

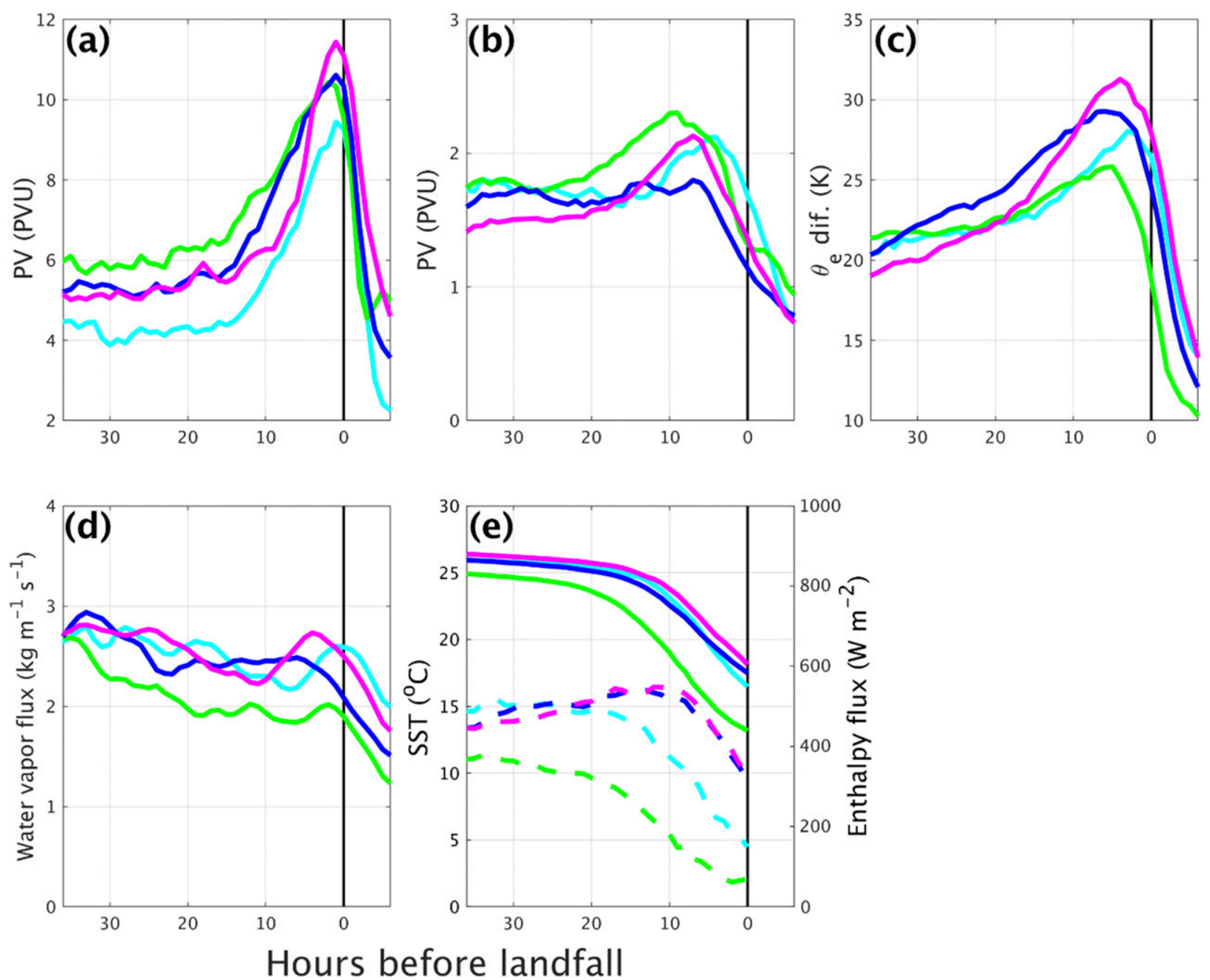

FIG. 17. Cluster-mean evolution of (a) 900-hPa PV averaged from (a) 0-50 and (b) 100-200 km, (c) mean difference in 850-hPa $\theta_{e}$ between 0-50 and 200-400 km, (d) 850-200-hPa integrated vertical water vapor flux averaged from 0 to $250 \mathrm{~km}$, and (e) mean SST (solid lines) and ocean-air enthalpy flux (dashed lines) averaged from 0 to $250 \mathrm{~km}$. All cluster means are calculated relative to the landfall time of each ensemble member composing that cluster.

heat release from weaker convection likely contributes to more rapid warm-core decay; however, the green cluster's greater warm core decay is most prominent at low levels; at $500 \mathrm{hPa}$ the green cluster has a comparable $\theta_{e}$ anomaly at landfall to the three more westward clusters (Figs. 19e-h). This indicates that the greater warm core decay in the green cluster is primarily driven by lowertropospheric processes.

Slightly earlier Sandy-trough interaction and warm seclusion formation relative to landfall among green cluster members also contribute to greater lowertropospheric warm-core decay by landfall. Twelve hours before landfall, low- $\theta_{e}$ values have wrapped the farthest around the south side of Sandy in the green cluster and the connection to the high- $\theta_{e}$ reservoir southeast of Sandy is narrowest (cf. Figs. $15 \mathrm{~h}$ and $15 \mathrm{e}-\mathrm{g}$ ).
Furthermore, the 5560-m geopotential height contour of Sandy merges with the trough $20 \mathrm{~h}$ before landfall in the green composite, compared to $14-18 \mathrm{~h}$ before landfall in the other composites (not shown). The earlier Sandytrough interaction and warm seclusion formation relative to landfall among green cluster members allows more time for the warm seclusion to contract and decay before landfall.

An earlier (relative to landfall) and less intense Sandy-trough interaction, along with lower SSTs, suppresses prelandfall intensification in the green cluster and yields a marginally weaker storm at landfall compared to the other clusters. During the $24 \mathrm{~h}$ prior to landfall, green members deepen by a mean of $5.6 \mathrm{hPa}$, compared to $10.1-12.2 \mathrm{hPa}$ in the other three clusters. However, a substantial bifurcation in intensification rate 

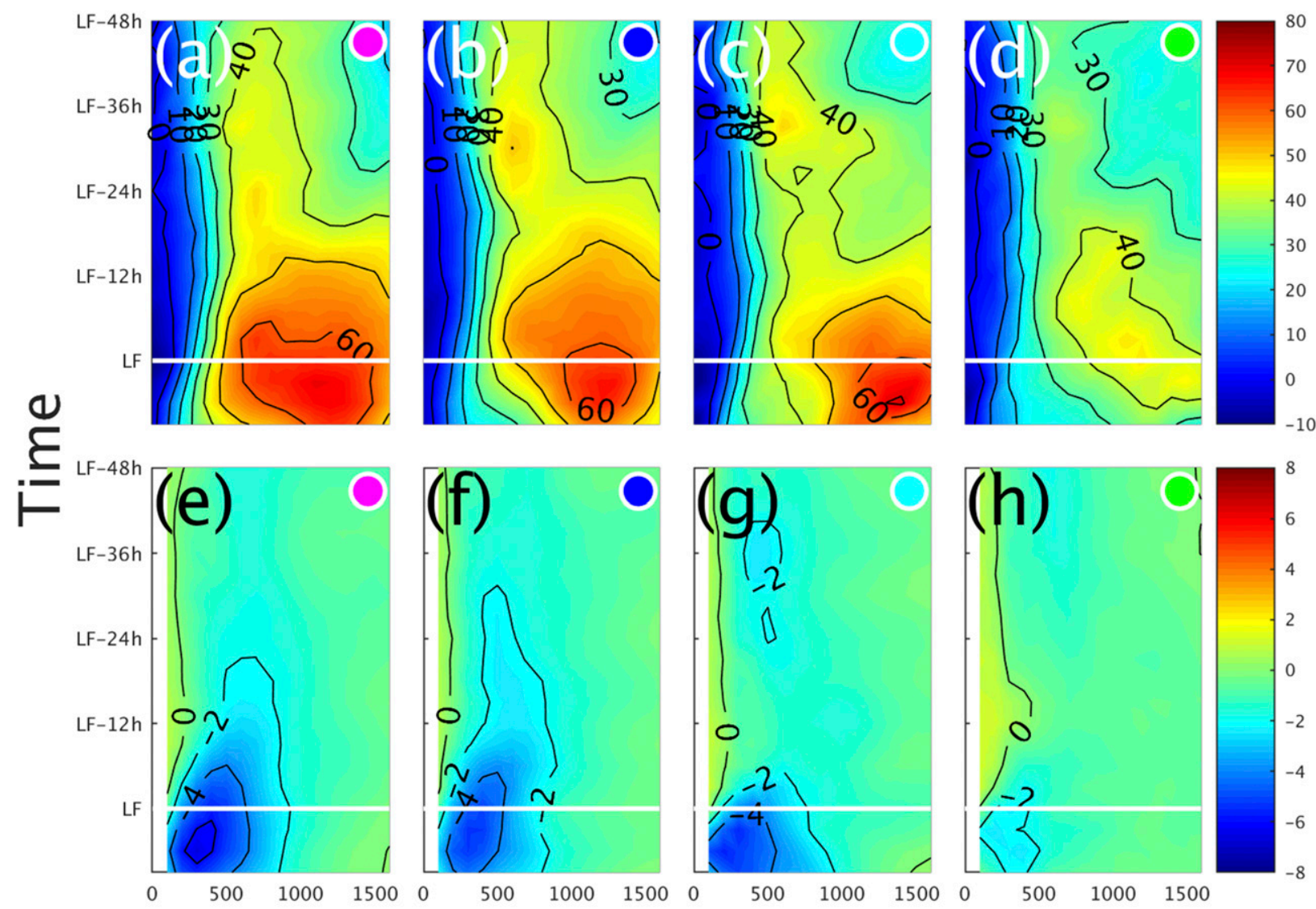

Radial distance $(\mathrm{km})$

FIG. 18. Cluster-mean, azimuthally averaged (a)-(d) 200-300-hPa EMFC ( $\mathrm{m} \mathrm{s}^{-1}$ day $^{-1}$ ) and (e)-(h) 850-hPa radial wind speed (m s ${ }^{-1}$ ) relative to the landfall time of each ensemble member. The landfall time is indicated by white lines. The landfall time of each simulation is approximated as the last 3-hourly model output time before landfall.

occurs among the green members; three deepen by at least $12 \mathrm{hPa}$ during the $24 \mathrm{~h}$ before landfall, while four intensify little or weaken slightly. Thus, in some green members the intensification during the warm seclusion stage is comparable to the more westward clusters, while other members experience little or no intensification. At landfall, the green cluster has a mean minimum central pressure of $943 \mathrm{hPa}$, slightly higher than the more westward clusters $(938-940 \mathrm{hPa})$. Green members also have the lowest mean maximum $10-\mathrm{m}$ wind speed during the six hours prior to landfall $\left(31.8 \mathrm{~m} \mathrm{~s}^{-1}\right.$; cf. $34.0-$ $35.1 \mathrm{~m} \mathrm{~s}^{-1}$ in the other three clusters), and have the smallest region of wind speeds exceeding $25.7 \mathrm{~m} \mathrm{~s}^{-1}$ $(50 \mathrm{kt})$ shortly before landfall (Figs. 20a-d).

Although the green cluster has the most decayed warm seclusion at landfall, seclusion intactness does not increase monotonically from east to west among the clusters. The cyan cluster has the highest mean $0-50-\mathrm{km}$ $850-\mathrm{hPa} \theta_{e}$ at landfall $(344.2 \mathrm{~K})$, statistically significantly higher ( $95 \%$ confidence; two-tailed $t$ test) than the blue cluster $(337.4 \mathrm{~K})$ and marginally higher than magenta $(342.3 \mathrm{~K})$. The eastward track of the cyan cluster relative to magenta and blue, and the farther east mean landfall location (Fig. 9a) result in a slightly weaker and later (relative to landfall) trough interaction. Mean 200-300$\mathrm{hPa}$ EMFC values exceeding $50 \mathrm{~m} \mathrm{~s}^{-1} \mathrm{day}^{-1}$ occur farther from Sandy's center and later relative to landfall in the cyan composite compared to the magenta and blue composites (cf. Figs. 18c and 18a,b).

A less intense Sandy-trough interaction in the cyan cluster produces a weaker 850 -hPa gradient compared to the magenta and blue clusters (Fig. 17c; cf. Figs. 15k and $15 \mathrm{i}, \mathrm{j}$ ) and weaker $850-\mathrm{hPa}$ radial inflow before landfall (cf. Figs. 18g and 18e,f). Weaker radial inflow is consistent with slower warm seclusion contraction. However, unlike green members, cyan members move over a similar mean SST to magenta and blue members (solid lines in Fig. 17e), allowing greater maintenance of the lower-troposphere warm core compared to the green cluster. 


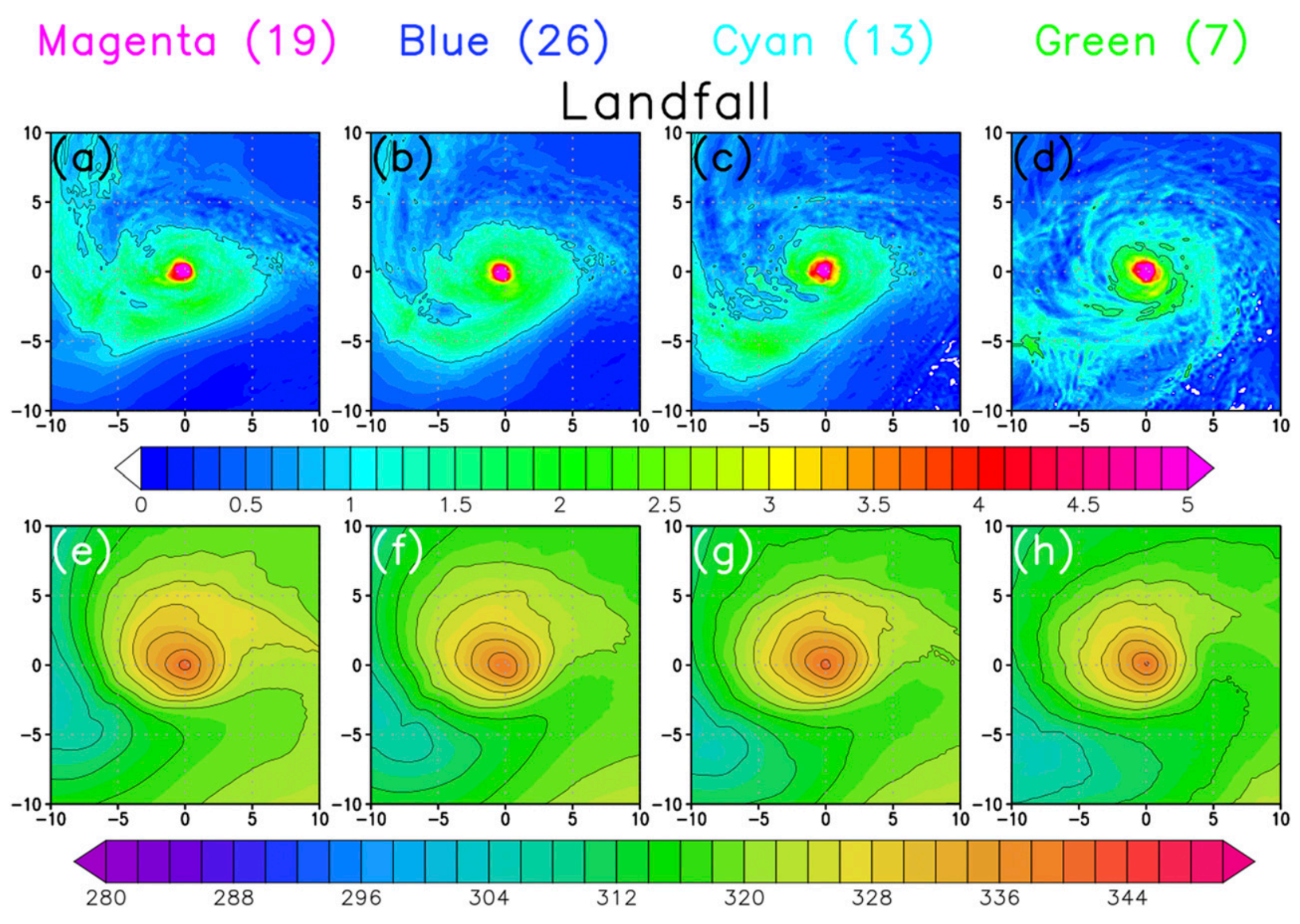

FIG. 19. Cluster composites of (a)-(d) 500-hPa potential vorticity (PVU; shaded and contoured at 1 PVU) and (e)-(h) 500-hPa equivalent potential temperature $(\mathrm{K}$; shaded and contoured every $4 \mathrm{~K})$ at the landfall time of each ensemble member.

The slightly later storm-trough interaction and weaker radial inflow in the cyan cluster delay the concentration of low-level PV at Sandy's center relative to landfall. In each cluster, PV concentrates at $0-50-\mathrm{km}$ radii (Fig. 17a) as it begins to decrease at larger radii (e.g., 100-200 km; Fig. 17b). In the cyan cluster, mean 900-hPa PV in the 100-200-km annulus peaks four hours before landfall, three to five hours later than in the other three clusters, and remains highest through landfall (Fig. 17b). Less prelandfall warm seclusion contraction and PV concentration in the cyan cluster yield a larger region of intense winds. Three hours before landfall, the cyan composite has the largest region of winds exceeding $25.7 \mathrm{~m} \mathrm{~s}^{-1}(50 \mathrm{kt})$, along with a more expansive warm seclusion than the blue or magenta clusters (Fig. 20).

Compared to the magenta composite, the blue composite shows greater warm seclusion decay at landfall (cf. Figs. $15 \mathrm{n}$ and $15 \mathrm{~m}$ ); mean $0-50-\mathrm{km} 850-\mathrm{hPa} \theta_{e}$ is $4.9 \mathrm{~K}$ lower at landfall. The later landfall time of the blue cluster, compared to magenta, is associated with earlier landfall-relative storm-trough interaction. Six hours before landfall, the 2-PVU contour in the blue composite
(Fig. 16j) has advanced farther east along the south side of Sandy compared to the magenta composite (Fig. 16i). This earlier trough interaction causes slightly earlier landfall-relative warm seclusion formation, contraction, and decay. The mean $850-\mathrm{hPa} \theta_{e}$ difference between 0-50 and $200-400 \mathrm{~km}$ peaks six hours before landfall in the blue cluster, versus four hours in magenta (Fig. 17c). While these timing differences do not result in substantially different wind fields as Sandy approaches landfall (cf. Figs. 20a and 20b), the magenta composite shows a more intense precipitation shield at landfall (as measured by $850-\mathrm{hPadBZ}$; cf. Figs. 20e and 20f). This result accords with the findings of Munsell and Zhang (2014), which found that earlier Sandy-trough interaction relative to landfall yielded less precipitation over land.

\section{Summary and conclusions}

The relationship between the track of Hurricane Sandy and its structural evolution during extratropical transition is examined using an ensemble of 72 regional convection-permitting WRF simulations. Simulations 

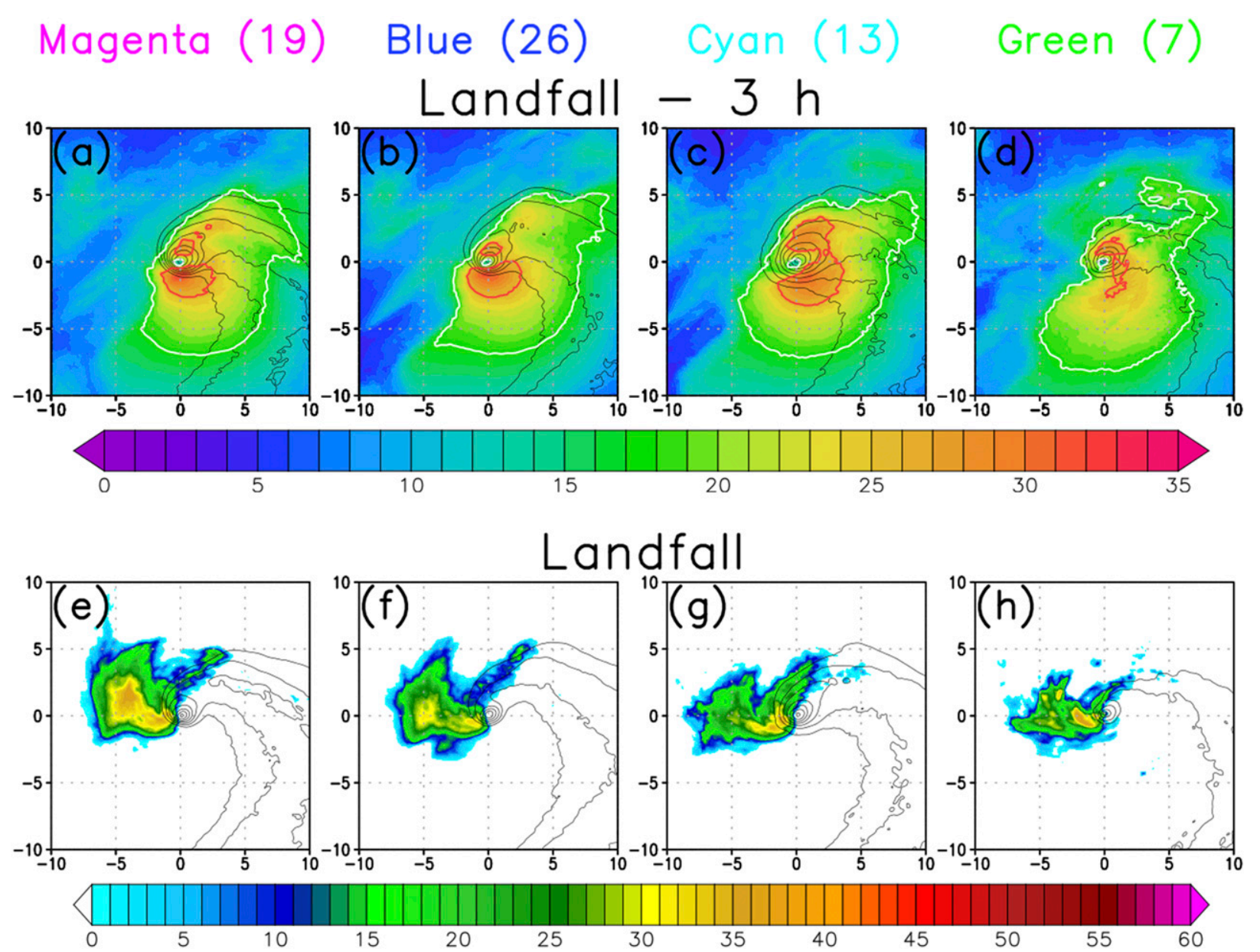

FIG. 20. Cluster composites of (a)-(d) $10-\mathrm{m}$ sustained wind speed $\left(\mathrm{m} \mathrm{s}^{-1}\right.$; shaded with white contours at $17.5 \mathrm{~m} \mathrm{~s}^{-1}$ and red contours at $\left.25.7 \mathrm{~m} \mathrm{~s}^{-1}\right) 3 \mathrm{~h}$ before landfall and (e)-(h) $850-\mathrm{hPa} \mathrm{dBZ}$ at the time of landfall. Equivalent potential temperature at $850 \mathrm{hPa}$ is contoured every $4 \mathrm{~K}$ between 316 and $344 \mathrm{~K}$ in each panel.

use initial and boundary conditions from ECMWF-IFS and NCEP-GEFS ensemble forecasts initialized at 0000 UTC 25 October 2012 and span the period from 0000 UTC 27 October to 0000 UTC 1 November. Storm tracks and CPS paths from the WRF simulations between 0000 UTC 27 October and 0000 UTC 31 October are partitioned using regression mixture models. A sixcluster solution with fourth- (fifth) order polynomials is selected for track (CPS) clustering. Cluster-mean values and composites of the four most populous track clusters are examined to elucidate how Sandy's structural evolution varies with track in standard and landfall-relative frameworks.

The clearest differences among the four analyzed clusters concern the timing of the interaction between Sandy and the midlatitude trough and extratropical transition completion. The Sandy-trough interaction (as measured by upper-level eddy momentum flux convergence and potential vorticity advection) occurs earliest in the westernmost cluster and progressively later in more eastward clusters. These timing differences in Sandy-trough interaction manifest in corresponding differences in the timing of PV wrap-up, warm seclusion formation, and seclusion contraction and decay. In the westernmost magenta cluster warm seclusion formation and decay occur approximately $30 \mathrm{~h}$ earlier than the easternmost green cluster.

While ET timing varies substantially among clusters, the ET timing and process are remarkably similar when examined in a landfall-relative framework. In each cluster, PV from the trough descends and wraps around Sandy shortly before landfall. In concert with this wrap-up, cool lower-tropospheric air partially surrounds Sandy's core, increasing radial baroclinicity and forming a short-lived warm seclusion. As the warm seclusion contracts before landfall, lower-tropospheric PV is concentrated at small radii. Thus, among the members of each cluster, Sandy makes landfall with a small, contracting 
warm seclusion and an intense, low-level PV core. These results indicate the high predictability of the warm seclusion ET of Sandy before landfall.

Although the synoptic-scale ET process is similar among the four clusters examined, subtle, but relevant, intercluster differences exist in the Sandy-trough interaction and storm structural evolution prior to landfall. These are especially evident when contrasting the easternmost green cluster with the three more westward clusters. In the green cluster Sandy undergoes a less intense storm-trough interaction, and the resulting warm seclusion is weaker (as measured by the $850-\mathrm{hPa}$ radial $\theta_{e}$ gradient). High 850 -hPa $\theta_{e}$ values in Sandy's core also decay the most before landfall in the green cluster; lower sea surface temperatures and slightly earlier (landfall relative) trough interaction likely contribute to this greater erosion. In the green cluster Sandy also undergoes the least mean intensification during the $24 \mathrm{~h}$ prior to landfall, though there is substantial variability among members.

The cyan cluster has the most intact warm seclusion at landfall, though it tracks east of the magenta and blue clusters and makes landfall later. Cyan members' more eastward track and landfall location keep Sandy farther from the trough axis and delay low-level PV concentration near Sandy's center relative to landfall. This contributes to cyan members retaining a more expansive region of damaging winds as Sandy approaches landfall. Members of the blue and magenta clusters take similar tracks, but in blue members Sandy interacts with the trough slightly earlier relative to landfall; this produces greater warm seclusion decay and a less intense precipitation shield at landfall. This difference in precipitation fields is consistent with that found by Munsell and Zhang (2014) between their "GOOD" and "FAIR" composite groups, which take similar tracks to magenta and blue cluster members, respectively.

Despite the intercluster differences described above, the simulations composing each of the four most populous clusters show Sandy developing a warm seclusion that contracts before landfall, concentrating lowertropospheric PV at small radii. It remains to be determined why the landfall-relative timing of Sandy's warm seclusion development is so similar among ensemble members. The large size of both Sandy and the midlatitude trough may have minimized the impact of track variations on the synoptic-scale interaction. Furthermore, even in simulations in which Sandy moves out to sea and does not interact with the trough (the orange cluster; Fig. 9a), a cyclonic Rossby wave breaking event occurs. Thus, the occurrence of cyclonic wave breaking, which favors PV wrap-up and warm seclusion development, shows low sensitivity to variations in Sandy's track.
Although cyclonic wave breaking occurs among members of all clusters, it appears to occur later in simulations in which Sandy moves out to sea. Cyclones can facilitate cyclonic wave breaking through negative PV advection by the divergent wind (e.g., Molinari et al. 1995; Galarneau et al. 2013). Results from this study show greater negative PV advection in more westward clusters. We hypothesize that a westward (eastward) track generates more (less) forcing for cyclonic wave breaking, and thus earlier (later) PV wrap-up and warm seclusion formation. However, a westward (eastward) track makes landfall earlier (later); thus, the structure of Sandy at landfall is similar.

The ET process of Sandy and its structure at landfall do not differ drastically among clusters, but this will not necessarily be true for other TCs that threaten landfall while undergoing ET. The method of cluster analysis presented here may prove useful for forecasting future ET events that pose landfall hazards. Objective clustering of ensemble forecasts, and analysis of storm characteristics in each cluster, would allow forecasters to determine in real time how storm intensity and structural evolution vary with storm track. It would also provide a tool for forecasters and decision-makers to examine the most probable landfall hazards associated with each potential storm track.

Acknowledgments. This work was funded by the National Science Foundation under Grant ATM-1322532. We thank Linus Magnusson of ECMWF for providing the IFS simulations employed in this study. We also thank Casey Webster for providing the compositing code, and David Vollaro for providing the code to calculate momentum fluxes. We are grateful to Juan Jesus González Alemán for helpful discussions and feedback on this work. We appreciate the able assistance of Chuck Pavloski and Chad Bahrmann in database analysis and management. Finally, we thank three anonymous reviewers for their thoughtful comments, which substantially improved the manuscript.

\section{REFERENCES}

Arnott, J. M., J. L. Evans, and F. Chiaromonte, 2004: Characterization of extratropical transition using cluster analysis. Mon. Wea. Rev., 132, 2916-2937, https://doi.org/10.1175/MWR2836.1.

Blake, E. S., T. B. Kimberlain, R. J. Berg, J. P. Cangialosi, and J. L. Beven II, 2013: Tropical Cyclone Report Hurricane Sandy (22-29 October 2012). Tech. Rep. AL182012, National Hurricane Center, 157 pp., https://www.nhc.noaa.gov/data/tcr/ AL182012_Sandy.pdf.

Demuth, J., M. DeMaria, and J. A. Knaff, 2006: Improvement of Advanced Microwave Sounder Unit tropical cyclone intensity and size estimation algorithms. J. Appl. Meteor. Climatol., 45, 1573-1581, https://doi.org/10.1175/JAM2429.1. 
ECMWF, 2011: ECMWF's Operational Model Analysis, starting in 2011. Research Data Archive at the National Center for Atmospheric Research, Computational and Information Systems Laboratory, accessed 30 March 2018, https://doi.org/ 10.5065/D6ZG6Q9F.

- 2017: Changes in ECMWF model. ECMWF, accessed 17 March 2017, http://www.ecmwf.int/en/forecasts/documentationand-support/changes-ecmwf-model.

Evans, J. L., and R. E. Hart, 2003: Objective indicators of the life cycle evolution of extratropical transition for Atlantic tropical cyclones. Mon. Wea. Rev., 131, 909-925, https://doi.org/10.1175/ 1520-0493(2003)131<0909:OIOTLC>2.0.CO;2.

Gaffney, S. J., A. W. Robertson, P. Smith, S. J. Camargo, and M. Ghil, 2007: Probabilistic clustering of extratropical cyclones using regression mixture models. Climate Dyn., 29, 423-440, https://doi.org/10.1007/s00382-007-0235-z.

Galarneau, T. J., C. A. Davis, and M. A. Shapiro, 2013: Intensification of Hurricane Sandy (2012) through extratropical warm core seclusion. Mon. Wea. Rev., 141, 4296-4321, https:// doi.org/10.1175/MWR-D-13-00181.1.

Hanley, D., J. Molinari, and D. Keyser, 2001: A composite study of the interactions between tropical cyclones and uppertropospheric troughs. Mon. Wea. Rev., 129, 2570-2584, https:// doi.org/10.1175/1520-0493(2001)129<2570:ACSOTI>2.0.CO;2.

Hart, R. E., 2003: A cyclone phase space derived from thermal wind and thermal asymmetry. Mon. Wea. Rev., 131, 585-616, https://doi.org/10.1175/1520-0493(2003)131<0585:ACPSDF $>$ 2.0.CO;2.

- J. L. Evans, and C. Evans, 2006: Synoptic composites of the extratropical transition life cycle of North Atlantic tropical cyclones: Factors determining posttransition evolution. Mon. Wea. Rev., 134, 553-578, https://doi.org/10.1175/MWR3082.1.

Hoskins, B., 1997: A potential vorticity view of synoptic development. Meteor. Appl., 4, 325-334, https://doi.org/10.1017/ S1350482797000716.

— the storm of 15-16 October 1987. Weather, 43, 122-129, https:// doi.org/10.1002/j.1477-8696.1988.tb03890.x.

Hubert, L., and P. Arabie, 1985: Comparing partitions. J. Classif., 2, 193-218, https://doi.org/10.1007/BF01908075.

Kimball, S. K., and J. L. Evans, 2002: Idealized numerical simulations of hurricane-trough interaction. Mon. Wea. Rev., 130, 2210-2227, https://doi.org/10.1175/1520-0493(2002)130<2210: INSOHT $>2.0 . \mathrm{CO} ; 2$.

Klein, P., P. A. Harr, and R. L. Elsberry, 2002: Extratropical transition of western North Pacific tropical cyclones: Midlatitude and tropical cyclone contributions to reintensification. Mon. Wea. Rev., 130, 2240-2259, https://doi.org/10.1175/15200493(2002)130<2240:ETOWNP>2.0.CO;2.

Kofron, D. E., E. A. Ritchie, and J. S. Tyo, 2010: Determination of a consistent time for the extratropical transition of tropical cyclones. Part II: Potential vorticity metrics. Mon. Wea. Rev., 138, 4344-4361, https://doi.org/10.1175/2010MWR3181.1.

Kowaleski, A. M., and J. L. Evans, 2016: Regression mixture model clustering of multimodel ensemble forecasts of hurricane
Sandy: Partition characteristics. Mon. Wea. Rev., 144, 38253846, https://doi.org/10.1175/MWR-D-16-0099.1.

Kuruppumullage Don, P., J. L. Evans, F. Chiaromonte, and A. M. Kowaleski, 2016: Mixture-based path clustering for synthesis of ECMWF ensemble forecasts of tropical cyclone evolution. Mon. Wea. Rev., 144, 3301-3320, https://doi.org/10.1175/ MWR-D-15-0214.1.

Leroux, M.-D., M. Plu, and F. Roux, 2016: On the sensitivity of tropical cyclone intensification under upper-level trough forcing. Mon. Wea. Rev., 144, 1179-1202, https://doi.org/ 10.1175/MWR-D-15-0224.1.

Magnusson, L., J.-R. Bidlon, S. T. K. Lang, A. Thorpe, N. Wedi, and M. Yamaguchi, 2014: Evaluation of medium-range forecasts for Hurricane Sandy. Mon. Wea. Rev., 142, 1962-1981, https://doi.org/10.1175/MWR-D-13-00228.1.

Marchok, T. P., 2002: How the NCEP tropical cyclone tracker works. Preprints, 25th Conf. on Hurricanes and Tropical Meteorology, San Diego, CA, Amer. Meteor. Soc., P1.13, https://ams.confex. com/ams/25HURR/techprogram/paper_37628.htm.

Milrad, S. M., E. A. Atallah, and J. R. Gyakum, 2009: Dynamical and precipitation structures of poleward-moving tropical cyclones in eastern Canada, 1979-2005. Mon. Wea. Rev., 137, 836-851, https://doi.org/10.1175/2008MWR2578.1.

Molinari, J., S. Skubis, and D. Vollaro, 1995: External influences on hurricane intensity. Part III: Potential vorticity structure. J. Atmos. Sci., 52, 3593-3606, https://doi.org/10.1175/15200469(1995)052<3593:EIOHIP>2.0.CO;2.

Munsell, E. B., and F. Zhang, 2014: Prediction and uncertainty of Hurricane Sandy (2012) explored through a real-time cloudpermitting ensemble analysis and forecast system assimilating airborne Doppler radar observations. J. Adv. Model. Earth Syst., 6, 38-58, https://doi.org/10.1002/2013MS000297.

NCEI, 2018: Global Ensemble Forecast System (GEFS). NOAA/NCEI, accessed 30 March 2018, https://www.ncdc. noaa.gov/data-access/model-data/model-datasets/globalensemble-forecast-system-gefs.

Rand, W. M., 1971: Objective criteria for the evaluation of clustering methods. J. Amer. Stat. Assoc., 66, 846-850, https://doi.org/ 10.1080/01621459.1971.10482356.

Ritchie, E. A., and R. L. Elsberry, 2003: Simulations of the extratropical transition of tropical cyclones: Contributions by the midlatitude upper-level trough to reintensification. Mon.Wea. Rev., 131, 2112-2128, https://doi.org/10.1175/1520-0493(2003) 131,2112:SOTETO.2.0.CO;2.

$\longrightarrow$, and - 2007: Simulations of the extratropical transition of tropical cyclones: Phasing between the upper-level trough and tropical cyclones. Mon. Wea. Rev., 135, 862-876, https:// doi.org/10.1175/MWR3303.1.

Skamarock, W. C., and Coauthors, 2008: A description of the Advanced Research WRF version 3. NCAR Tech. Note NCAR/ TN-475+STR, 113 pp., https://doi.org/10.5065/D68S4MVH.

Torn, R. D., J. S. Whitaker, P. Pegion, T. M. Hamill, and G. J. Hakim, 2015: Diagnosis of the source of GFS medium-range track errors in Hurricane Sandy (2012). Mon. Wea. Rev., 143, 132-152, https://doi.org/10.1175/MWR-D-14-00086.1. 